\title{
Hardy spaces and analytic continuation
} of Bergman spaces

\author{
Bulletin de la S. M. F., tome 126, no 3 (1998), p. 435-482 \\ $<$ <ttp://www.numdam.org/item?id=BSMF_1998_126_3_435_0>
}

(C) Bulletin de la S. M. F., 1998, tous droits réservés.

L'accès aux archives de la revue «Bulletin de la S. M. F. » (http: //smf.emath.fr/Publications/Bulletin/Presentation.html) implique l'accord avec les conditions générales d'utilisation (http://www.numdam.org/ conditions). Toute utilisation commerciale ou impression systématique est constitutive d'une infraction pénale. Toute copie ou impression de ce fichier doit contenir la présente mention de copyright.

\section{Numdam}




\title{
HARDY SPACES AND ANALYTIC CONTINUATION OF BERGMAN SPACES
}

\author{
BY Wolfgang BERTRAM AND JOACHIM HILGERT $(*)$
}

\begin{abstract}
We introduce a family of weighted Bergman spaces associated to a compactly causal symmetric space and investigate the relation of the corresponding Hardy space with the analytic continuation of this family. For an important class of compactly causal symmetric spaces defined by involutions of Euclidian Jordan algebras we obtain precise results which are analogous to the corresponding situation for bounded symmetric domains.

RÉSUMÉ. - ESPACES DE HARDY ET PROLONGEMENT ANALYTIQUE DES ESPACES DE Bergman. - Nous introduisons une famille d'espaces de Bergman pondérés associée à un espace symétrique compactement causal, et nous examinons la relation entre l'espace de Hardy correspondant et le prolongement analytique de cette famille. Pour une classe importante d'espaces symétriques compactement causaux, définie par des involutions d'algèbres de Jordan euclidiennes, nous obtenons des résultats précis qui sont en analogie avec la situation correspondante pour les domaines bornés symétriques.
\end{abstract}

\section{Introduction}

\subsection{A family of weighted Bergman spaces.}

Let $D$ be a bounded symmetric domain of tube type, realized as a generalized disc in the complexification $V_{\mathbb{C}}$ of a Euclidian Jordan algebra $V$. There is a well known family of Hilbert spaces of holomorphic functions on $D$, called weighted Bergman spaces and defined by

$$
\begin{aligned}
& B_{\mu}^{2}(D):=\left\{\begin{array}{r}
f \in(D) \\
\end{array}\right. \\
&\left.\|f\|_{\mu}^{2}:=c_{\mu} \int_{D}|f(z)|^{2}(\operatorname{Det} B(z, \bar{z}))^{\mu-1} \mathrm{~d} z<\infty\right\}
\end{aligned}
$$

(*) Texte reçu le 14 avril 1998 , accepté le $1^{\text {er }}$ juillet 1998.

W. Bertram, J. Hilgert, Mathematisches Institut, Technische Universität Clausthal, Erzstr. 1, D-38678 Clausthal-Zellerfeld (Deutschland).

Email: bertram@math.tu-clausthal.de, hilgert@math.tu-clausthal.de.

AMS classification: 22 E 45, 32 M 15, 32 A 35.

Keywords: Hardy spaces, Bergman space, Jordan algebra. 
( $c f$. [FK94, Ch. XIII]). Here the density appearing in the integral is defined by the Bergman polynomial

$$
B(z, w):=\operatorname{id}_{V_{\mathbb{C}}}-2 z \square w+P(z) P(w),
$$

where $x \square y$ and $P(x)$ are related to the triple product

$$
T(x, y, z)=x(y z)-y(x z)+(x y) z
$$

associated to the Jordan algebra $V_{\mathbb{C}}$ via

$$
T(x, y, z)=(x \square y) z \quad \text { and } \quad T(x, y, x)=P(x) y \text {, }
$$

and the constant $c_{\mu}$ is chosen such that, if the norm $\|1\|_{\mu}$ of the constant function 1 is finite, then it is equal to one. If $V$ is simple, then the space $B_{\mu}^{2}(D)$ is not reduced to zero if and only if

$$
\mu>1-\frac{r}{2 n}
$$

(with $n=\operatorname{dim} V$ and $r$ the rank of $V$; $c f$. [FK94]; there the parameter is $\nu=\mu \cdot 2 n / r)$. The space $B_{1}^{2}(D)$ is the ordinary Bergman space of $D$. The harmonic analysis of the weighted Bergman spaces with respect to the group $G(D)$ of holomorphic automorphisms of $D$ (or, more generally, its universal covering $\widetilde{G}(D)$ ) is well developed. These spaces contribute to the holomorphic discrete series of $G(D)$.

In this work we will introduce an additional structure, define a more general class of weighted Bergman spaces and study some natural problems in their harmonic analysis. The additional structure is given by distinguishing an open dense domain

$$
\Xi:=\left\{z \in D ; f_{\alpha}(z) \neq 0\right\}
$$

in $D$, where $\alpha$ is an involutive automorphism of $V$ and $f_{\alpha}$ the holomorphic polynomial on $V$ given by

$$
f_{\alpha}(z):=\operatorname{Det}(P(z+\alpha(z))) .
$$

For example, if $V=V_{1} \times V_{1}$ is the direct product of a simple Jordan algebra with itself and

$$
\alpha((x, y))=(y, x)
$$

then $\Xi$ is the domain considered by Chadli [Cha98]. Other data lead to the cases considered by Koufany-Ørsted [KØ97], Molchanov [Mo97], and Betten-Ólafsson [BO98].

$$
\text { TOME } 126-1998-\mathrm{N}^{\circ} 3
$$


We now define a family of weighted Bergman spaces depending on two real parameters $\mu$ and $\lambda$ :

$$
\begin{aligned}
& B_{\mu, \lambda}^{2}(\Xi):=\{f \in \mathcal{O}(\Xi) ; \\
&\left.\|f\|_{\mu, \lambda}^{2}:=c_{\mu} \int_{\Xi}|f(z)|^{2}\left|f_{\alpha}(z)\right|^{-\lambda}(\operatorname{Det} B(z, \bar{z}))^{\mu-1} \mathrm{~d} z<\infty\right\},
\end{aligned}
$$

where $\mathrm{d} z$ is Lebesgue measure, restricted to the open domain $\Xi \subset D$. For $\lambda=0$, any function from $B_{\mu, \lambda}^{2}$ can be extended to a holomorphic function on $D$ (Prop. 2.1.1), and thus $B_{\mu, 0}^{2}(\Xi) \cong B_{\mu}^{2}(D)$. In the second parameter, the behavior is "periodic" since clearly for $k \in \mathbb{N}$ multiplication by $f_{\alpha}^{k}$ yields an isomorphism of $B_{\mu, \lambda}^{2}$ onto $B_{\mu, \lambda+2 k}^{2}$. The argument remains valid for any rational parameter $k=p / q$ such that there exists a holomorphic function $f$ with $f=f_{\alpha}^{k}$ (i.e. $\left.f^{q}=\left(f_{\alpha}\right)^{p}\right)$; we call such parameters admissible.

The weighted Bergman spaces are Hilbert spaces such that for all $z \in \Xi$ the point evalutions $f \mapsto f(z)$ are continuous. Thus there exists a vector $K_{z}^{(\mu, \lambda)}$ such that $f(z)=\left(f \mid K_{z}^{(\mu, \lambda)}\right)$. The function

$$
K^{(\mu, \lambda)}: \Xi \times \Xi \longrightarrow \mathbb{C}, \quad(w, z) \longmapsto K_{z}^{(\mu, \lambda)}(w)
$$

is called the reproducing kernel of $B_{\mu, \lambda}^{2}(\Xi)$. We prove ( $c f$. Cor. 2.1.4):

TheOREM 1. - If $\mu>1-\frac{r}{2 n}$ and $\frac{\lambda}{2}$ is an admissible parameter, then the space $B_{\mu, \lambda}^{2}(\Xi)$ is non-trivial, and its reproducing kernel is given by

$$
K^{(\mu, \lambda)}(z, w)=f_{\alpha}(z)^{\lambda / 2}(\operatorname{Det} B(z, \bar{w}))^{-\mu}{\overline{f_{\alpha}(w)}}^{\lambda / 2},
$$

where $B$ is given by (0.2) and $f_{\alpha}$ by $(0.4)$.

Next we explain how harmonic analysis enters into this picture.

\subsection{Group actions and spectrum of weighted Bergman spaces.}

As is well known, the group $G(D)$ acts transitively on $D$. The domain $\Xi \subset D$ is stable under the action of the subgroup of $G(D)$

$$
\begin{aligned}
G: & =G(D)^{(-\alpha)_{*}} \\
& :=\left\{g \in G(D) ;(-\alpha)_{*} g:=(-\alpha) \circ g \circ(-\alpha)=g\right\},
\end{aligned}
$$

but this action is not transitive. However, the action of $G$ on the open dense subset

$$
X:=\left\{u \in \Sigma ; f_{\alpha}(u) \neq 0\right\}
$$


of the Shilov boundary

$$
\Sigma:=\left\{z \in V_{\mathbb{C}} ;(\bar{z})^{-1}=z\right\}
$$

of $D$ is transitive. We write $X=G / H$, where $H$ is the stabilizer of the base point $o=i e \in X$. Then $X$ is a compactly causal symmetric space, and $\Xi$ is the domain in its complexification $X_{\mathbb{C}}$ considered in [HOØ91]

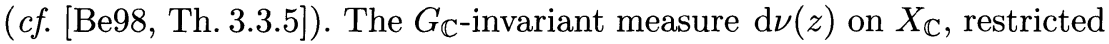
to $\Xi$, is related to the Lebesgue measure $\mathrm{d} z$ of $V_{\mathbb{C}}$ via

$$
\mathrm{d} \nu(z)=\left|f_{\alpha}(z)\right|^{-1} \mathrm{~d} z
$$

(cf. [Be98, Prop. 2.4.3]). Thus the Bergman space of $\Xi$

$$
B^{2}(\Xi):=\left\{f \in \mathcal{O}(\Xi) ; \int_{\Xi}|f(z)|^{2} \mathrm{~d} \nu(z)<\infty\right\}
$$

is equal to the space $B_{1,1}^{2}(\Xi)$. The other spaces corresponding to the "diagonal" $\lambda=\mu$ have the following interpretation:

THEOREM 2. - If $\frac{1}{2}$ is an admissible parameter, then the reproducing kernel of $B^{2}(\Xi)$ is given by the function $K^{(1,1)}$ defined in Theorem 1 . If $\frac{1}{2} \mu$ is an admissible parameter and $\mu>1-r /(2 n)$, then the space

$$
B_{\mu}^{2}(\Xi):=\left\{f \in \mathcal{O}(\Xi) ; \int_{\Xi}|f(z)|^{2}\left(K^{(1,1)}(z, \bar{z})\right)^{1-\mu} \mathrm{d} \nu(z)<\infty\right\}
$$

is non-trivial and equal to $B_{\mu, \mu}^{2}(\Xi)$. Its reproducing kernel is

$$
K^{(\mu, \mu)}=\left(K^{(1,1)}\right)^{\mu} .
$$

The group $G$ acts unitarily on the spaces $B_{\mu}^{2}(\Xi)$ via ordinary translation of functions. We are interested in describing the decomposition of $B_{\mu}^{2}(\Xi)$ into irreducible representations. There are two approaches to this problem: the first describes the representations appearing in the decomposition as highest weight representations and then determines the corresponding highest weights; this has been worked out for the group cases and $\mu=1$ by Krötz ( $c f$. [Kr97]). The second approach, which we use in this work, is geometric. We apply an idea that appears in the work of Jakobsen and Vergne [JV79] to the specific geometric situation given by a bounded symmetric domain with an involution $\alpha$ : The space

$$
D^{-}:=\{z \in D ; \alpha(z)=-z\} \subset D
$$

TOME $126-1998-\mathrm{N}^{\circ} 3$ 
is a bounded symmetric domain in its own right; it is associated to $X=G / H$ in the sense that $D^{-}=G / K$ with $K$ maximal compact in $G$. It is a special feature of our setup (not predicted by the general theory of the domain $\Xi$ in [HOØ91]) that $D^{-}$belongs to the boundary of $\Xi$; this boundary is in a sense "opposite" to the boundary $X \subset \partial \Xi$. Elements of $B_{\mu, 2 k}^{2}(\Xi)(k \geq 0$ admissible) have a holomorphic continuation to all of $D$; thus we can restrict them and all their partial derivatives to $D^{-}$. These differential restrictions determine the function entirely, and because $G$ acts transitively on $D^{-}$, it is not too difficult to decompose the space of restrictions thus obtained under the action of $G$ (Theorem 2.3.5). The interest of this technique lies in the fact that it yields not only the spectrum, but also exhibits natural intertwining operators of certain function spaces into the holomorphic discrete series of $G$.

\subsection{Analytic continuation of weighted Bergman spaces and classical Hardy spaces.}

The reproducing kernel of a Hilbert space is a positive kernel, corresponding to the positivity of the scalar product. Conversely, every positive kernel defines a Hilbert space of functions. For $\mu>1-r /(2 n)$, the function $K^{(\mu, 0)}(w, z)$ given by the formula in Theorem 1 is the reproducing kernel of a weighted Bergman space and is thus positive. The set $\mathcal{W} \subset \mathbb{R}$ of parameters $\mu$ for which the formula in Theorem 1 defines a positive definite kernel $K^{(\mu, 0)}$ (the so called Wallach set) can be determined explicitly, cf. [FK94, Th. XIII.2.7]:

$$
\left.\mathcal{W}=\left\{0, \frac{n-r}{2(r-1) n}, \ldots, \frac{1}{2}-\frac{r}{2 n}\right\} \cup\right] \frac{1}{2}-\frac{r}{2 n}, \infty[.
$$

It is strictly bigger than the Bergman range $\mu>1-r /(2 n)$ belonging to the weighted Bergman spaces. The corresponding "absract" reproducing kernel spaces will be denoted by $B_{\mu}(D)$, the superscript 2 being reserved for $\mu$ in the Bergman range. The question arises whether the Hilbert spaces $B_{\mu}(D)$ belonging to parameters outside the Bergman range have an analytic significance similar to the Bergman spaces. This is in fact true for the parameter $\mu=\frac{1}{2}$ : The space $B_{\frac{1}{2}}(D)$ is the Hardy space $H^{2}(D)$ of holomorphic functions $f$ on $D$ having square integrable boundary values in the sense that

$$
\|f\|_{H^{2}(D)}^{2}:=\sup _{0<r<1} \int_{\Sigma}|f(r u)|^{2} \mathrm{~d} \sigma(u)
$$

is finite, where $\mathrm{d} \sigma$ is the normalized $U$-invariant measure on $\Sigma$ ( $c f$. [FK94, p. 269]). Here $U$ is the stabilizer of 0 in $G(D)$ which is compact and acts 
transitively on $\Sigma$. The space $H^{2}(D)$ has a reproducing kernel, the CauchySzegö kernel $S(z, u)$, defined by the property

$$
f(z)=\int_{\Sigma} S(z, u) f(u) \mathrm{d} \sigma(u)
$$

Since $H^{2}(D)=B_{\frac{1}{2}}(D)$, we have the explicit formula $S=K^{\left(\frac{1}{2}, 0\right)}$ given by Theorem 1 for the Cauchy-Szegö kernel. Its square is the reproducing kernel of the classical Bergman space $B_{1}^{2}(D)$.

\subsection{The Hardy space of $\Xi$ and its comparison with the classical one.}

We are now going to explain to what extent the preceding results can be generalized to the domain $\Xi$. The Hardy space $H^{2}(\Xi)$ of $\Xi$ is defined as the space of holomorphic functions $f$ on $\Xi$ having square integrable boundary values on $X$ in the sense that

$$
\|f\|_{H^{2}(\Xi)}^{2}:=\sup _{\gamma \in \Gamma} \int_{X}|f(\gamma \cdot x)|^{2} \mathrm{~d} x<\infty
$$

where $\Gamma$ is a certain subsemigroup in the complex group $G_{\mathbb{C}}$ such that $\Xi=\Gamma \cdot i e$. An explicit formula for the reproducing kernel of $H^{2}(\Xi)$ is known only in some special cases. Our approach to this problem is trying to realize the space $H^{2}(\Xi)$ as the point belonging to the parameter $\mu=\frac{1}{2}$ in the analytic continuation of the weighted Bergman spaces $B_{\mu}^{2}(\Xi)$. Note that because of Theorem 2 the analytic continuation of the spaces $B_{\mu}^{2}(\Xi)$ is easy to describe: These are just the spaces $B_{\mu, \mu}(\Xi)$ corresponding to the kernel $K^{(\mu, \mu)}$ for admissible parameters $\mu$ in the Wallach set $\mathcal{W}$. Our main results in this context are:

Theorem 3. - Assume that $\frac{1}{4}$ is an admissible parameter. Then we have an inclusion of function spaces

$$
B_{\frac{1}{2}}(\Xi) \subset H^{2}(\Xi)
$$

Theorem 4. - Assume that $X$ is irreducible. Then the inclusion (0.13) is an equality if and only if the rank of $X$ is equal to its split rank; this is the case if and only if $X$ admits both compact and non-compact Cartan subspaces. Then the Cauchy-Szegö kernel of $H^{2}(\Xi)$ is given by $K^{\left(\frac{1}{2}, \frac{1}{2}\right)}$, and its square is the Bergman kernel $K^{(1,1)}$ of $\Xi$.

In the situation of Theorem 4, multiplication by $f_{\alpha}^{\frac{1}{4}}$ is an isomorphism

$$
H^{2}(D)=B_{\frac{1}{2}}(D) \longrightarrow B_{\frac{1}{2}, \frac{1}{2}}(\Xi),
$$

TOME $126-1998-\mathrm{N}^{\circ} 3$ 
and in this sense $H^{2}(D)$ is realized as a subspace of $H^{2}(\Xi)$. The question whether in this way the classical and the non-classical Hardy spaces are isomorphic has attracted much interest during the last years, $c f . e . g$. [BO98], [Cha98], [KØ97], [OØ98]. From our point of view, the problem can the Hardy space be realized as the point with parameter $\mu=\frac{1}{2}$ in the analytic continuation of the weighted Bergman spaces? is the mathematically correct formulation of the question are the classical and the non-classical Hardy spaces isomorphic? - the trouble being that "non-canonical" isomorphisms between the two Hardy-spaces may exist, as shows the compact case (Section 3.4). Moreover, our formulation of the problem makes sense for any compactly causal symmetric space, not only for the ones related to Jordan algebras. However, our proof of Theorem 4 uses the Jordan-structure via the explicit formula for the Bergman kernel. It would be interesting to have an abstract proof not involving this formula, either geometric or via highest weight theory. From a geometric point of view it is remarkable that in the cases where $(0.13)$ is an equality, the "causal group" $G(D)$ has a unitary and irreducible representation in $H^{2}(\Xi)$.

\section{Contents of the paper}

1. Geometric and algebraic preliminaries

2. The spectrum of a family of weighted Bergman spaces

3. Comparison of Hardy spaces

4. Open problems

Section 1 contains the classification (Section 1.5) and some preliminary results on involutions of (Euclidian) Jordan algebras which are, however, of interest in their own right ( $c f$. Theorem 1.6.1, Theorem 1.8.2). The full information provided by Chapter 1 is needed only for the proof of Theorem 4; in the other parts of Chapters 2 and 3 only the general facts explained in Sections 1.1-1.4 are used. Finally, the approach to Bergmanand Hardy spaces presented in this paper leads naturally to some open problems (Chapter 4) which we will investigate in subsequent work.

We would like to thank M. Chadli and J. Faraut for helpful discussions and the Mathematics Institute of Jussieu, where part of this work was done, for the kind hospitality.

The first named author gratefully acknowledges support by the DFGgrant HI $4125-1$.

BULLETIN DE LA SOCIÉTÉ MATHÉMATIQUE DE FRANCE 


\section{Geometric and algebraic preliminaries}

\subsection{Euclidian Jordan algebras and associated symmetric domains.}

Let $V$ be a Euclidian Jordan algebra with unit element $e$ and $\Omega$ the associated symmetric cone. The cone $\Omega$ can be defined as the component of $e$ of the set of invertible elements of $V$. Our basic reference is [FK94]; we follow the notation introduced there. The tube domain

$$
T_{\Omega}=V+i \Omega \subset V_{\mathbb{C}}
$$

has, via the Cayley transform

$$
C: D \rightarrow T_{\Omega}, \quad C(z)=i(e+z)(e-z)^{-1}
$$

a bounded realization $D=C^{-1}\left(T_{\Omega}\right)$. The Shilov boundary

$$
\Sigma=\left\{z \in V_{\mathbb{C}} ; \bar{z}=z^{-1}\right\}
$$

of $D$ is, via the Cayley transform, equivalent to the conformal compactification $V^{c}$ of $V$ introduced in [Be96], and the group $G\left(T_{\Omega}\right)$ of biholomorphic automorphisms of $T_{\Omega}$, acting by birational maps on $V$, is (up to connected components) the conformal group $\mathrm{Co}(V)$ introduced in [Be96]. Its complexification $G\left(T_{\Omega}\right)_{\mathbb{C}}$ is the conformal group $\mathrm{Co}\left(V_{\mathbb{C}}\right)$ of $V_{\mathbb{C}} ;$ it acts by complex birational maps on $V_{\mathbb{C}}$. The stabilizer $U$ of the base point 0 in $G(D)$ is a compact group acting linearly on $V_{\mathbb{C}}$. Its complexification is (up to connected components) the structure group $\operatorname{Str}\left(V_{\mathbb{C}}\right)$ of $V_{\mathbb{C}}$. The group $U$ acts transitively on $\Sigma$.

It is well known that the $\operatorname{disc} D$ is the connected component of 0 of the set

$$
\left\{z \in V_{\mathbb{C}} ; \operatorname{Det} B(z, \bar{z}) \neq 0\right\},
$$

where $B$ is as in (0.2). The $G(D)$-invariant measure on $D$ has the density (Det $B(z, \bar{z}))^{-1}$ with respect to the Lebesgue measure of $V_{\mathbb{C}}$, where the determinant is taken over $\mathbb{C}(c f .[\mathrm{FK} 94])$.

\subsection{A decomposition of bounded symmetric domains.}

Let $\alpha$ be an involution (automorphism of order 2) of the Euclidian Jordan algebra $V$ and denote by the same letter its $\mathbb{C}$-linear extension to $V_{\mathbb{C}}$. Then we have the eigenspace-decompositions $V=V^{+} \oplus V^{-}$and $V_{\mathbb{C}}=V_{\mathbb{C}}^{+} \oplus V_{\mathbb{C}}^{-}$with respect to $\alpha$. The intersections of the eigenspaces with the bounded symmetric domain $D$,

$$
D^{+}:=D \cap V_{\mathbb{C}}^{+}, \quad D^{-}:=D \cap V_{\mathbb{C}}^{-},
$$

TOME $126-1998-\mathrm{N}^{\circ} 3$ 
are easily seen to be bounded symmetric domains ( $c f$. [Be98, Prop. 3.1.1]), the former being of tube type (since $V^{+}$is a Euclidian Jordan algebra), the latter in general not. Their automorphism groups are related to the group $G(D)$ as follows. Let $\phi_{*}(g):=\phi g \phi^{-1}$ be the conjugation by an element $\phi$ of $\operatorname{Co}\left(V_{\mathbb{C}}\right)$; then $G(D)$ is stable under the involutions $\alpha_{*}$ and $(-\alpha)_{*}$, and we denote the connected fixed point groups of these involutions by

$$
G^{+}:=G(D)_{o}^{\alpha_{*}}, \quad G^{-}:=G(D)_{o}^{(-\alpha)_{*}} .
$$

It is then easily verified that $G^{+}$acts transitively on $D^{+}$and $G^{-}$acts transitively on $D^{-}$; in other words, we have surjective homomorphisms

$$
\rho_{ \pm}: G^{ \pm} \longrightarrow G\left(D^{ \pm}\right), \quad g \longmapsto g_{\mid D^{ \pm}} .
$$

In the "generic case" these homomorphisms are also injective ( $c f$. Remark 1.8.5). The case $\alpha=\mathrm{id}_{V}$ is somewhat singular since $V^{-}$is then reduced to a point. Note that in this case $G^{-}$is the compact linear group $U$ defined in Section 1.1, whereas $G\left(D^{-}\right)$should be defined as the trivial group.

\subsection{Compactly causal Makarevič spaces.}

Let, as above, $\alpha$ be an involution of the Euclidian Jordan algebra $V$ and $G:=G^{-}$. Then, according to [Be98, Th. 3.3.6], the group $G$ acts transitively on the open dense domain

$$
X=\left\{u \in \Sigma ; f_{\alpha}(u) \neq 0\right\}
$$

of the Shilov boundary $\Sigma$. Further, $X$ is a symmetric space with geodesic symmetry $-j$ with respect to the base point $i e$, where $j(z)=z^{-1}$ is the inverse in the Jordan algebra $V_{\mathbb{C}}$. The corresponding involution of the group $G$ is given by

$$
(-j)_{*} g=(-j) g(-j) .
$$

We thus can write $X=G / H$ with $H$ open in $G^{(-j)_{*}}$.

The space $X$ is a causal symmetric space: The symmetric cone $\Omega$ defines on $V$ a flat causal structure (a constant field of cones obtained by translating $\Omega$ to each tangent space of $V$ ) which is transported to the Shilov boundary $\Sigma$ via the Cayley-transform $C$. The structure on $V$ is invariant under the group $\operatorname{Co}(V)=G\left(T_{\Omega}\right)$, and therefore the structure on $\Sigma$ is invariant under $G(D)$; in particular, its restriction to $X$ is invariant under $G$. We describe this causal structure on $X$ in more detail: Let $\tau$ be the complex conjugation of $V_{\mathbb{C}}$ with respect to $V$. Then $(-j \tau)_{*}$ is a Cartan involution on $G$ (cf. [Be98, Section 2.2]). Denote by $\mathfrak{g}=\mathfrak{k} \oplus \mathfrak{p}$ the corresponding Cartan decomposition of $\mathfrak{g}$. Moreover 
let $\mathfrak{g}=\mathfrak{h} \oplus \mathfrak{q}$ be the decomposition associated to the involution $(-j)_{*}$. These decompositions can be described as spaces of vector fields. We denote by $j_{*} v$ the homogeneous quadratic vector field $\left(j_{*} v\right)(x)=-P(x) v$ with $v \in V$ and by $\mathfrak{s t r}\left(V_{\mathbb{C}}\right) \subset \mathfrak{g l}\left(V_{\mathbb{C}}\right)$ the Lie algebra of the structure group of $V_{\mathbb{C}}$. Then using the fact that $\mathfrak{g}$ is the subalgebra of $\mathfrak{c o}\left(V_{\mathbb{C}}\right)$ fixed under $(-\alpha)_{*}$ and under $(j \tau)_{*}$, one gets

$$
\left\{\begin{array}{l}
\mathfrak{p}=\left\{v+j_{*} \tau v ; v \in V_{\mathbb{C}}^{-}\right\} \cong V_{\mathbb{C}}^{-}, \\
\mathfrak{k}=i L\left(V^{+}\right) \oplus \operatorname{Der}(V)^{\alpha_{*}}, \\
\mathfrak{q}=\mathfrak{q}_{\mathfrak{k}} \oplus \mathfrak{q}_{\mathfrak{p}}=i L\left(V^{+}\right) \oplus\left\{v+j_{*} v ; v \in V^{-}\right\}, \\
\mathfrak{h}=\mathfrak{h}_{\mathfrak{k}} \oplus \mathfrak{h}_{\mathfrak{p}}=\operatorname{Der}(V)^{\alpha_{*}} \oplus i\left\{v-j_{*} v ; v \in V^{-}\right\} .
\end{array}\right.
$$

The cone $W$ in the tangent space $T_{0} X \cong \mathfrak{q}$ defining the causal structure on $X$ is given by

$$
\begin{aligned}
W:=\mathrm{d} C(0)^{-1} \Omega=-\left\{i L\left(v^{+}\right)+\right. & \left(v^{-}+j_{*} v^{-}\right) \\
& \left.v \in \Omega, v=v^{+}+v^{-}, v^{ \pm} \in V^{ \pm}\right\} .
\end{aligned}
$$

In fact, the Cayley transformed version of the cone $W \subset \mathfrak{q}$ is the cone of vector fields

$$
W^{\prime}:=C_{*} W=\left\{v-j_{*} \alpha v ; v \in \Omega\right\}
$$

in the space of vector fields

$$
\mathfrak{q}^{\prime}:=C_{*} \mathfrak{q}=\left\{v-j_{*} \alpha v ; v \in V\right\}
$$

where $C_{*}$ denotes the forward transport of vector fields by the Cayley transform $C$. This cone corresponds to the causal structure considered in [Be98, Section 3.2].

In particular,

$$
W \cap \mathfrak{q}_{\mathfrak{k}}=-i\left\{L\left(v^{+}\right) ; v^{+} \in \Omega \cap V^{+}\right\}
$$

is isomorphic to $\Omega^{+}:=\Omega \cap V^{+}$. Note that this is just the symmetric cone associated to $V^{+}$(this holds because $\Omega^{+}=e^{L\left(V^{+}\right)} \cdot e$ ). Thus the causal structure we consider has the property that $W \cap \mathfrak{q}_{\mathfrak{k}} \neq \emptyset$; i.e. $X$ is compactly causal (cf. [HO96, Def. 3.1.8]), and moreover it has the special feature that both $W$ and $W \cap \mathfrak{q}_{\mathfrak{k}}$ are self-dual (w.r.t. suitable scalar products).

Finally, note that the space $X$ appears as an open orbit in a Shilov boundary. Not all compactly causal symmetric spaces have this property,

TOME $126-1998-\mathrm{N}^{\circ} 3$ 
but "most" of them have (see Section 1.5). These are precisely the Makarevič spaces (cf. [Be96], [Be98]) among the compactly causal symmetric spaces.

\subsection{The domain $\Xi$ associated to a compactly causal Makarevič space.}

The domain $\Xi$ defined by equation (0.3) is related to the holomorphic action of a complex semigroup as follows. If $W \subset \mathfrak{q}$ is the $\operatorname{Ad}(H)$-invariant cone given by (1.2), we denote by $\exp (i W)$ the image of $i W \subset i \mathfrak{q} \subset \mathfrak{g}_{\mathbb{C}}$ under the exponential map of $G_{\mathbb{C}}$. Then, according to [Be98, Th. 3.3.5],

$$
\Xi=G \exp (i W) \cdot i e .
$$

In [HOØ91] the domain $\Xi$ is introduced via an extension of the cone $W$ to an $\operatorname{Ad}(G)$-invariant cone $\widetilde{W}$ in $\mathfrak{g}$ : by general results on invariant cones (cf. [HO96]) there exists a $G$-invariant open convex cone $\widetilde{W} \subseteq \mathfrak{g}$ which satisfies

$$
(-j)_{*}(\widetilde{W})=-\widetilde{W} \text { and } \widetilde{W} \cap \mathfrak{q}=W .
$$

Then ( $c f$. [HOØ91, Lemma 3.1 and its proof]) $G \exp (i \widetilde{W})$ is a subsemigroup of $G_{\mathbb{C}}$ such that $\Xi=G \exp (i \widetilde{W}) \cdot i e$. It should be noted here that the extension $\widetilde{W}$ of $W$ with the given properties is in general not unique. Our results will not depend on the extension $\widetilde{W}$ chosen. However, there is one "biggest" extension which is rather canonical in the given geometric context and which we will fix in the sequel.

Proposition 1.4.1. - Let $S(D):=\left\{g \in \operatorname{Co}\left(V_{\mathbb{C}}\right) ; g(D) \subset D\right\}$ be the compression semigroup of $D$ and let

$$
\Gamma:=G_{\mathbb{C}} \cap S(D)=\left\{g \in G_{\mathbb{C}} ; g(D) \subset D\right\} .
$$

Then $\Gamma$ is a semigroup with $\Xi=\Gamma \cdot$ ie, and it contains $G \exp (i W)$. It is of the form $\Gamma=G \exp (i \widetilde{W})$, where $\widetilde{W}$ is a regular $\operatorname{Ad}(G)$-invariant cone in $\mathfrak{g}$ with

$$
(-j)_{*}(\widetilde{W})=-\widetilde{W} \text { and } \widetilde{W} \cap \mathfrak{q}=W .
$$

Proof. - From [Be98, Cor. 3.3.3] it follows via Cayley-transform that $G \exp (i W) \subset \Gamma$. Therefore [Be98, Th. 3.3.5] implies that $\Xi \subset \Gamma \cdot$ ie. The other inclusion follows from the fact that $\Gamma \subset\left(G_{\mathbb{C}} \cap S(D)\right)$ and thus $\Gamma \cdot$ ie $\subset\left(X_{\mathbb{C}} \cap D\right)$ which is, according to [Be98, Th. 2.1.3 (iv)], equal to $\Xi$.

The remaining statements are best proved using the tube realization $T_{\Omega}$ of $D$. Then the compression semigroup $S\left(T_{\Omega}\right)$ is of the form

$$
S\left(T_{\Omega}\right)=G\left(T_{\Omega}\right) \exp \left(i C_{\max }\right)
$$


with the maximal invariant cone $C_{\max } \subset \mathfrak{g}\left(T_{\Omega}\right)$ given by

$$
C_{\max }=\left\{X \in \mathfrak{g}\left(T_{\Omega}\right) ;(\forall v \in V) X(v) \in \Omega\right\}
$$

(see [Cha98, Th. 1.1]). From Equations (1.3) and (1.4) we deduce that

$$
W^{\prime}=\mathfrak{q}^{\prime} \cap C_{\max }
$$

Moreover, the Cayley transformed version of the involution $(-j)^{*}$ is $(-\mathrm{id})_{*}$, and the description of $C_{\max }$ given above shows that

$$
(-\mathrm{id})_{*} C_{\max }=-C_{\max }
$$

It follows that the cone $\widetilde{W}:=C_{\max } \cap \mathfrak{g}$ has all the desired properties. (Note, however, that we cannot conclude that this cone is maximal in $\mathfrak{g}$.

\subsection{Classification.}

In this section we classify simple pairs $(V, \alpha)$; this means that $V$ is a Euclidian Jordan algebra having no proper ideal invariant under the involution $\alpha$. The spaces $V$ and $V^{+}$are described as Jordan algebras; in particular, Sym and Herm denote Jordan algebras of symmetric, respectively Hermitian, matrices with their natural Jordan product

$$
X \cdot Y=\frac{1}{2}(X Y+Y X)
$$

and $\mathbb{R}^{p} \times \mathbb{R}^{q}$ is the space $\mathbb{R}^{p+q}$ with the Jordan product

$$
x \cdot y=b(x, e) y+b(y, e) x-b(x, y) e
$$

where $b$ is a form of signature $(p, q)$ and $e$ such that $b(e, e)=1$. The spaces $V^{-}$are described as Jordan triple-systems (JTS): they inherit from $V$ the Jordan triple product

$$
(x, y, z) \longmapsto x(y z)-y(x z)+(x y) z
$$

which defines $V^{-}$as non-degenerate JTS in the sense of [Sa80, I.6]. In particular, Asym and Aherm denote JTS of skew-symmetric, resp. skewHermitian matrices and $\operatorname{Mat}(p \times q ; \mathbb{F})$ denotes the JTS of $p \times q$-matrices over F. By Hermitian we mean, if there is no other specification: Hermitian with respect to the canonical involution of the base field ( $c f$. [Be96]). In the case $\mathbb{F}$ is the skew field $\mathbb{H}$ of quaternions, we consider also its involution

$$
\varphi: 1 \mapsto 1, \quad i \mapsto-i, \quad j \mapsto j, \quad k \mapsto-k
$$

TOME $126-1998-\mathrm{N}^{\circ} 3$ 
Then the Jordan algebra $\operatorname{Herm}(m, \varphi, \mathbb{H})$ is isomorphic to the Jordan triple system $\operatorname{Aherm}(m, \mathbb{H}), c f$. [Be96, 1.2.1]. Finally,

$$
J=\left(\begin{array}{cc}
0 & I \\
-I & 0
\end{array}\right) \text { and } I_{p, q}=\left(\begin{array}{cc}
I_{p} & 0 \\
0 & -I_{q}
\end{array}\right)
$$

where $I_{n}$, or simply $I$ if the size is clear from the context, is the identity matrix of size $n \times n$.

1.5.1 Table of simple pairs $(V, \alpha)$ (cf. [Ma73] and [Be96]).

I) $V=\operatorname{Herm}(m, \mathbb{C}), V_{\mathbb{C}}=\operatorname{Mat}(m \times m, \mathbb{C}), \operatorname{Co}(V)_{o} \cong \mathrm{SU}(m, m), \frac{n}{r}=m$.

1) $\alpha(Z)=I_{p, q} Z I_{p, q}(p+q=m)$,

$$
X \cong \mathrm{U}(p, q) \text {, }
$$

$V^{+}=\operatorname{Herm}(p, \mathbb{C}) \oplus \operatorname{Herm}(q, \mathbb{C}), V^{-} \cong \operatorname{Mat}(p \times q ; \mathbb{C})$,

$f_{\alpha}(Z)=\operatorname{Det}\left(Z+I_{p, q} Z I_{p, q}\right)^{2 m}$.

2) $\alpha(Z)=Z^{t}$,

$X \cong \mathrm{SO}^{*}(2 m) / \mathrm{SO}(m, \mathbb{C})$,

$V^{+}=\operatorname{Sym}(m, \mathbb{R}), V^{-}=i \operatorname{Asym}(m, \mathbb{R})$,

$f_{\alpha}(Z)=\operatorname{Det}\left(Z+Z^{t}\right)^{2 m}$.

3) $\alpha(Z)=J Z^{t} J^{-1}(m=2 k$ even $)$,

$X \cong \operatorname{Sp}(2 k, \mathbb{R}) / \operatorname{Sp}(k, \mathbb{C})$,

$V^{+} \cong \operatorname{Herm}(k, \mathbb{H}), V^{-} \cong \operatorname{Aherm}(k, \mathbb{H})$,

$f_{\alpha}(Z)=\operatorname{Det}\left(Z-J Z^{t} J\right)^{4 k}$.

II) $V=\operatorname{Sym}(m, \mathbb{R}), V_{\mathbb{C}}=\operatorname{Sym}(m, \mathbb{C}), \operatorname{Co}(V)_{o} \cong \operatorname{Sp}(m, \mathbb{R}), \frac{n}{r}=\frac{m+1}{2}$.

1) $\alpha(Z)=I_{p, q} Z I_{p, q}(p+q=m)$

$X \cong \mathrm{U}(p, q) / \mathrm{O}(p, q)$,

$V^{+}=\operatorname{Sym}(p, \mathbb{R}) \oplus \operatorname{Sym}(q, \mathbb{R}), V^{-} \cong \operatorname{Mat}(p \times q ; \mathbb{R})$,

$f_{\alpha}(Z)=\operatorname{Det}\left(Z+I_{p, q} Z I_{p, q}\right)^{m+1}$.

2) $\alpha(Z)=J Z J^{-1}(m=2 k$ even $)$,

$X \cong \operatorname{Sp}(k, \mathbb{R})$,

$V^{+} \cong \operatorname{Herm}(k, \mathbb{C}), V^{-} \cong \operatorname{Sym}(k, \mathbb{C})$,

$f_{\alpha}(Z)=\operatorname{Det}(Z-J Z J)^{2 k+1}$.

III) $V=\operatorname{Herm}(m, \mathbb{H}), V_{\mathbb{C}}=\operatorname{Sym}(J, \mathbb{C}), \operatorname{Co}(V)_{o} \cong \mathrm{SO}^{*}(4 m), \frac{n}{r}=2 m-1$.

1) $\alpha(Z)=I_{p, q} Z I_{p, q}(p+q=m)$,

$X \cong \mathrm{U}(2 p, 2 q) / \mathrm{Sp}(p, q)$, 


$$
\begin{array}{ll} 
& V^{+} \cong \operatorname{Herm}(p, \mathbb{H}) \oplus \operatorname{Herm}(q, \mathbb{H}), V^{-} \cong \operatorname{Mat}(p \times q ; \mathbb{H}), \\
& f_{\alpha}(Z)=\operatorname{Det}\left(Z+I_{p, q} Z I_{p, q}\right)^{2 m-1} . \\
2) & \alpha(Z)=\varphi(Z)\left(i . e .,(\varphi(Z))_{i j}=\left(\varphi\left(Z_{i j}\right) \text { with } \varphi \text { as above }\right)\right. \\
& X \cong \operatorname{SO}^{*}(2 m) \\
& V^{+} \cong \operatorname{Herm}(m, \mathbb{C}), V^{-} \cong \operatorname{Asym}(m, \mathbb{C}), \\
& f_{\alpha}(Z)=\operatorname{Det}\left(Z+Z^{t}\right)^{2 m-1} .
\end{array}
$$

IV) $V=\mathbb{R} \times \mathbb{R}^{n-1}, V_{\mathbb{C}}=\mathbb{C}^{n}, \mathrm{Co}(V)_{o} \cong \mathrm{SO}(2, n), \frac{n}{r}=\frac{n}{2}$.

1) $\alpha=\mathrm{id}_{V}$

$X \cong S^{n} \times S^{1}=\left(\mathrm{SO}(n) \times S^{1}\right) / \mathrm{SO}(n-1)$,

$f_{\alpha}(z)=\left(z_{1}+\cdots+z_{n}\right)^{2 n}$.

2) $\alpha=I_{p, n-p}, p>1$,

$X \cong(\mathrm{SO}(p) \times \mathrm{SO}(2, n-p) /(\mathrm{SO}(p-1) \times \mathrm{SO}(1, n-p))$,

$V^{+} \cong \mathbb{R} \times \mathbb{R}^{p-1}, V^{-} \cong \mathbb{R}^{n-p}$,

$f_{\alpha}(z)=\left(z_{1}+\cdots+z_{p}-z_{p+1}-\cdots-z_{n}\right)^{2 n}$.

3) $\alpha=I_{1, n-1}$,

$X \cong \mathrm{SO}(2, n-1) / \mathrm{SO}(1, n-1)$,

$V^{+} \cong \mathbb{R}, V^{-} \cong \mathbb{R}^{n-1}$,

$f_{\alpha}(z)=\left(z_{1}-\cdots-z_{n}\right)^{2 n}$.

V) $V=\operatorname{Herm}(3, \mathbb{O}), V_{\mathbb{C}}=\operatorname{Herm}\left(3, \mathbb{O}_{\mathbb{C}}\right)$,

$\mathrm{Co}(V)_{o} \cong E_{7(-25)}, \frac{n}{r}=9$.

1) $\alpha=\operatorname{id}_{V}$

$X \cong\left(E_{6(-14)} \times \mathrm{U}(1)\right) / F_{4(-20)}$,

$f_{\alpha}(Z)=\Delta(Z)^{18}$ (where $\Delta$ is the Jordan determinant).

(The Peirce-involution $\alpha(Z)=I_{1,2} Z I_{1,2}$ leads to the same space as $\alpha=\mathrm{id}_{V}$.)

2) $\alpha(Z)=\widetilde{Z}$ (the non-trivial involution of $\mathbb{O}$ ),

$X \cong \mathrm{SU}(6,2) / \mathrm{Sp}(3,1)$,

$V^{+}=\operatorname{Herm}(3, \mathbb{H}), V_{\mathbb{C}}^{-} \cong \operatorname{Mat}(6 \times 2 ; \mathbb{C})$,

$f_{\alpha}(Z)=\Delta(Z+\widetilde{Z})^{18}$

CT) (Cayley type): $V=V_{1} \times V_{1}$, where $V_{1}$ is one of the above Euclidian Jordan algebras, $\operatorname{Co}(V)_{o}=\mathrm{Co}\left(V_{1}\right)_{o} \times \operatorname{Co}\left(V_{1}\right)_{o}, P((x, y))=\left(\begin{array}{cc}P_{1}(x) & 0 \\ 0 & P_{1}(y)\end{array}\right)$, where $P_{1}$ is the quadratic representation of $V_{1}$.

$\alpha((x, y))=(y, x)$

TOME $126-1998-\mathrm{N}^{\circ} 3$ 
$V^{+} \cong V^{-} \cong V_{1}$

$f_{\alpha}((z, w))=\operatorname{Det} P((z+w, z+w))=\operatorname{Det} P_{1}(z+w)^{2}$, and

$X \cong \operatorname{Co}\left(V_{1}\right) / \operatorname{Str}\left(V_{1}\right)$. We obtain the following cases:

1) $X \cong \mathrm{SU}(n, n) /(\mathrm{Sl}(n, \mathbb{C}) \times \mathbb{R})$,

2) $X \cong \operatorname{Sp}(n, \mathbb{R}) / \mathrm{Gl}(n, \mathbb{R})$

3) $X \cong \mathrm{SO}^{*}(4 n) /(\mathrm{Sl}(n, \mathbb{H}) \times \mathbb{R})$,

4) $X \cong \mathrm{SO}(2, n) /(\mathrm{SO}(1, n-1) \times \mathbb{R})$,

5) $X \cong E_{7(-25)} /\left(E_{6(-26)} \times \mathbb{R}\right)$.

REMARK 1.5.2. - The following compactly causal symmetric spaces are not Makarevič spaces:

- the simple parts of the reductive spaces in the above list $(e . g . \mathrm{SU}(p, q)$ is the simple part of $\mathrm{U}(p, q))$,

- the spaces $\mathrm{SO}(2, p+q) /(\mathrm{SO}(1, p) \times \mathrm{SO}(1, q))$ with $\min (p, q)>1$,

- the group case $\mathrm{SO}(2, n)$ and

- some exceptional spaces ( $c f$. [HO96, p. 89]).

Conversely, the symmetric spaces $X$ from the above list which are not irreducible do not appear in the classification [HO96, p. 89] although their causal structure cannot be reduced to direct products (i.e. they are reducible as symmetric spaces, but irreducible as causal symmetric spaces; in [HO96] this distinction is not made, and a causal symmetric space is called irreducible if it is so as a symmetric space). These are the spaces I.- V.1 and IV.2. The latter case plays a rather exceptional role.

REMARK 1.5.3. - In all cases, the Jordan algebra $V^{+}$is either simple or a direct sum of two simple ideals. The latter happens precisely in the cases where $\alpha$ is a (non-trivial) Peirce-reflection, i.e. $\alpha=P(w)$ for some $w \in V(w \neq e)$ with $w^{2}=e(c f$. [Hw69, Lemma 3]). These are the cases I.1, II.1, III.1 (always $\alpha \neq \mathrm{id}_{V}$ ) and IV.2 $(p=2$ ).

REMARK 1.5.4. - The Jordan triple system (JTS) $V^{-}$is simple in all cases, but $V_{\mathbb{C}}^{-}$may be a direct sum of two simple JTS's. This happens if and only if $V^{-}$is in fact a complex JTS, and this corresponds exactly to the cases where $X$ is of group type, i.e.

(i) $X=(G \times G) / \operatorname{dia}(G \times G)$ with $G=\mathrm{U}(p, q)(\mathrm{I} .1)$,

(ii) $G=\operatorname{Sp}(k, \mathbb{R})($ II.2) or

(iii) $G=\mathrm{SO}^{*}(2 m)$ (III.2).

In these cases, $D^{-}$is a direct product of two isomorphic bounded symmetric domains. 
REMARK 1.5.5 (Admissible parameters). - Recall that a rational number $k=p / q$ is called an admissible parameter if there exists a holomorphic function $f$ on $\Xi$ with $f=f_{\alpha}^{k}$. Such a function is again constant in $V^{-}$-direction and is therefore determined by its restriction to $V^{+}$. Since the restriction $f_{\alpha \mid \Omega_{+}}$generates a one-dimensional space of functions under the action of $\operatorname{Str}\left(V^{+}\right)$, so will do the restriction $f_{\mid \Omega_{+}}$.

- If $V^{+}$is simple, it follows that $f_{\mid \Omega_{+}}$is a power of the Jordan determinant $\Delta_{+}$of $V^{+}$, i.e.

$$
f(x)=\left(\Delta_{+}(x+\alpha x)\right)^{\ell}
$$

with some integer $\ell$ ( $c f$. [FK94, Th. XII.2.2]). Thus the lowest admissible parameter $k$ is given by the condition

$$
f_{\alpha}(x)^{k}=\Delta_{+}(x+\alpha x) .
$$

Since $f_{\alpha}$ is homogeneous of degree $2 n$ and $\Delta_{+}$is homogeneous of degree $r_{+}$ (the rank of $V^{+}$), we get $k=r_{+} /(2 n)$ for the lowest admissible parameter if $V^{+}$is simple. (In the Cayley case this reduces to $k=r_{+} /\left(4 n_{+}\right)$. Note that $2 n_{+} / r_{+}$is always an integer, $c f$. Section 1.7.)

- If $V^{+}$is not simple (Peirce involutions), we write $V^{+}$as a sum of two simple algebras and apply the above argument to each term. Again we obtain that $k=r_{+} /(2 n)$ is the lowest admissible parameter. (Note that here $r_{+}=r$.)

We get the following list of admissible parameters in the cases corresponding to Table 1.5.1:

I.1. I.2. I.3. II.1. II.2. III.1. III.2. IV.1. IV.2. IV.3. V.1. V.2.

$\frac{\mathbb{Z}}{2 m} \frac{\mathbb{Z}}{2 m} \quad \frac{\mathbb{Z}}{8 k} \quad \frac{\mathbb{Z}}{m+1} \frac{\mathbb{Z}}{4 k+2} \frac{\mathbb{Z}}{2 m-1} \frac{\mathbb{Z}}{4 m-2} \quad \frac{\mathbb{Z}}{n} \quad \frac{\mathbb{Z}}{n} \quad \frac{\mathbb{Z}}{2 n} \quad \frac{\mathbb{Z}}{18} \quad \frac{\mathbb{Z}}{18}$

Later on it will be important to know whether $\frac{1}{2}$ and $\frac{1}{4}$ are admissible parameters. This can be read off the preceding table.

\subsection{Split and non-split involutions.}

In this section we prove in a classification free way that the involutions $\alpha$ given in the preceding section fall into two classes, called split and non-split.

Recall that the rank of a Euclidian Jordan algebra is the number of elements of a complete system of primitive orthogonal idempotents $c_{1}, \ldots, c_{r}$, also called a Jordan frame. The space

$$
R:=\bigoplus_{i=1}^{r} \mathbb{R} c_{i}
$$

TOME $126-1998-\mathrm{N}^{\circ} 3$ 
is a maximal associative subalgebra of $V$, and the space $L(R)$ is a Cartan subspace for the Cartan decomposition $\mathfrak{s t r}(V)=\operatorname{Der}(V) \oplus L(V)$, i.e. it is maximal abelian in $L(V)$. We can always find a Cartan subspace which is stable under a given involution, and correspondingly there is always a Jordan frame such that $\alpha(R)=R$ for a given involution $\alpha$ of $V$. Then we write $R=R^{+} \oplus R^{-}$for the corresponding decomposition of $R$ into eigenspaces of $\alpha$.

Theorem 1.6.1. - Let $V$ be a Euclidian Jordan algebra and $\alpha$ an involution such that $V$ has no proper $\alpha$-stable ideal. Then one can find a Jordan frame in $V$ such that:

(i) $\alpha(R)=R$,

(ii) the rank of the Euclidian Jordan algebra $V^{+}$is equal to $\operatorname{dim} R^{+}$,

(iii) either $R=R^{+}$or $\operatorname{dim} R^{+}=\operatorname{dim} R^{-}$.

Proof. - Let $e_{1}, \ldots, e_{r_{+}}$be a Jordan frame for the Euclidian Jordan algebra $V^{+}$. Since $e_{i}$ is an idempotent also in $V$, the eigenspace

$$
W_{i}:=V\left(e_{i}, 1\right):=\left\{x \in V ; L\left(e_{i}\right) x=x\right\}
$$

is a subalgebra of $V$ with unit element $e_{i}$ (see [FK94, Prop. IV.1.1]), and it inherits from $V$ a positive associative bilinear form, i.e. it is Euclidian. We claim that either $W_{i}=\mathbb{R} e_{i}$ or $W_{i}$ has rank two. In fact, $W_{i}$ is $\alpha$-stable; let

$$
W_{i}=W_{i}^{+} \oplus W_{i}^{-}
$$

be the corresponding decomposition. Since $e_{i}$ is primitive in $V^{+}$, it follows from the spectral theorem (see [FK94, Th. III.1.1]) that $V^{+}\left(e_{i}, 1\right)=W_{i}^{+}$ is one-dimensional. By the argument given before stating the theorem, we know that there exists a frame $w_{1}, \ldots, w_{\ell}$ in $W_{i}$ such that the vector space spanned by the $w_{j}$ 's is $\alpha$-stable. It follows that $\alpha$ permutes the elements of this frame. If $\operatorname{dim} W_{i}>1$, then $e_{i}=\sum_{j} w_{j}$ is not a primitive idempotent, and since $W_{i}^{+}=\mathbb{R} e_{i}$, it follows that no $w_{j}$ is fixed under $\alpha$. On the other hand, $w_{j}+\alpha\left(w_{j}\right)$ is an idempotent fixed under $\alpha$. It follows that already $w_{1}, \alpha\left(w_{1}\right)$ is a frame of $W_{i}$, and hence the rank of $W_{i}$ is 2 . We define

$$
f_{i}:=w_{1} .
$$

Next we are going to show that $f_{i}$ is actually primitive in $V$. To this end, let $e_{i}=\sum_{j} \lambda_{j} c_{j}$ be the spectral decomposition of $e_{i}$ in $V$ with respect to a Jordan frame $c_{1}, \ldots, c_{r}$ of $V$ (see [FK94, Th. III.1.2.]). Since $e_{i}$ is idempotent, it follows that $\lambda_{j}^{2}=\lambda_{j}$, whence $\lambda_{j}=1$ or $\lambda_{j}=0$. 
Because $e_{i} c_{j}=\lambda_{j} c_{j}$, this implies that those $c_{j}$ actually appearing in the decomposition $e_{i}=\sum_{j} \lambda_{j} c_{j}$ belong to $W_{i}$. But then they are also primitive idempotents in $W_{i}$, and since the rank of $W_{i}$ is at most two, there are at most two non-vanishing terms in the decomposition of $e_{i}$. If there is just one term, then $e_{i}$ is actually primitive in $V$. Otherwise we have $e_{i}=f_{i}+\alpha\left(f_{i}\right)$ with $f_{i}$ as above, and we conclude that $f_{i}$ is also primitive in $V$.

Now we can prove that

$$
\left\{f_{i}, \alpha f_{i}, e_{j} ; 1 \leq i, j \leq r_{+}, \operatorname{rk} W_{i}=2, \operatorname{rk} W_{j}=1\right\}
$$

is a frame in $V$ : it is clear from the construction that this is a complete system of idempotents which are primitive by what we have proved above. It only remains to be shown that they are orthogonal. But since the $e_{j}$ are orthogonal, we have for $i \neq j W_{i}=V\left(e_{i}, 1\right), W_{j} \subset V\left(e_{i}, 0\right)$, and since $V\left(e_{1}, 1\right) V\left(e_{j}, 0\right)=0$ (see [FK94, Prop. IV.1.1]), it follows that $W_{i} W_{j}=0$. We know already that $f_{i}$ and $\alpha\left(f_{i}\right)$ are orthogonal (if they are distinct). Together this implies that the idempotents in the system in question are orthogonal. It is clear that the vector space $R$ spanned by this system is $\alpha$-stable, and by construction $\operatorname{dim} R^{+}$is the rank of $V^{+}$. Thus we have established properties (i) and (ii).

In order to prove (iii) we assume first that $V^{+}$is simple. Then $\operatorname{Aut}\left(V^{+}\right)_{0}$ acts transitively on the set of primitive idempotents (see $\left[\right.$ FK94, Cor. IV.2.7]). Since the map $\operatorname{Aut}(V)_{o}^{\alpha_{*}} \rightarrow \operatorname{Aut}\left(V^{+}\right)_{o}, g \mapsto g_{\mid V^{+}}$is surjective ( $c f$. Prop. 1.8.1), it follows that any two of the $e_{i}$ are conjugate under $\operatorname{Aut}(V)^{\alpha_{*}}$, and therefore the corresponding $W_{i}$ are conjugate under this group. In particular, they have all the same rank. Thus in the frame of $V$ constructed above, either all idempotents are of the form $e_{j}$ or all appear in pairs $f_{i}, \alpha\left(f_{i}\right)$, and this implies (iii).

If $V^{+}$is not simple, then by a result of Helwig (see [Hw69, Lemma 3]), $\alpha$ is a Peirce-reflection $P(w)$ with respect to an idempotent $c$ of $V$, $w=2 c-e$. Then $V^{+}=V(c, 1) \oplus V(c, 0)(c f$. [FK94, p. 65]), and there is a frame of $V$ contained in $V^{+}$, i.e. $R=R^{+}$, and (iii) holds.

Definition 1.6.2. - Let $\alpha$ be an involution of $V$ and $R$ a frame having properties (i)-(iii) of the preceding theorem. Then, if $R=R^{+}$we say that $\alpha$ is split (w.r.t. $R$ ), and if $\operatorname{dim} R^{+}=\operatorname{dim} R^{-}$we say that $\alpha$ is non-split (w.r.t. $R$ ).

REMARK 1.6.3. - We specify which of the involutions from Table 1.5.1 are split and which are non-split. In the matrix cases we choose Jordan frames $c_{1}, \ldots, c_{r}$ such that $R=\sum_{i=1}^{r} \mathbb{R} c_{i}$ is the respective space of 
diagonal matrices. In the case of rank 2 (Type IV) we choose $c_{1}$ and $c_{2}$ such that they span the same space as the first two canonical base vectors of $\mathbb{R}^{n}$. The involutions $\alpha$ from Table 1.5.1 are either split or non-split for these Jordan frames. We distinguish further the cases that both $V$ and $V^{+}$are simple and that one of these algebras is not simple. Thus the involutions $\alpha$ are partitioned into four classes. Using the notation from Table 1.5.1, this partition looks as follows:

$$
V \text { and } V^{+} \text {simple } \quad V \text { or } V^{+} \text {not simple }
$$

$$
\begin{array}{ll}
\alpha \text { split } & \text { I.2, III.2, IV.2, V.2 Peirce involutions } \\
& \text { and } \alpha=\mathrm{id}_{V}
\end{array}
$$

$$
\alpha \text { non-split } \quad \text { I.3, II.2, IV.3 Cayley-type }
$$

Next we draw some consequences concerning the geometry of the associated compactly causal space $X$. Recall that the rank of a symmetric space $X$ is the dimension of a maximal abelian subspace of $\mathfrak{q}$ consisting of semisimple elements. Since our space $X$ is compactly causal, [HO96, Prop. 3.1.11(3)] together with $c$-duality shows that there is a compact Cartan subspace, i.e. a maximal abelian subspace contained in $\mathfrak{q}_{\mathfrak{k}}=\mathfrak{q} \cap \mathfrak{k}$. The split rank of $X$ is defined to be the dimension of a maximal abelian subspace of $\mathfrak{q}_{\mathfrak{p}}$.

Proposition 1.6.4.

(i) The rank of $X$ is equal to the rank of the Jordan algebra $V^{+}$, and the space $\mathfrak{a}:=i L\left(R^{+}\right)$is a compact Cartan subspace of $\mathfrak{q}$.

(ii) If $W \subset \mathfrak{q}$ is the invariant cone defined in Section 1.3 and $e_{1}, \ldots, e_{r^{+}}$the Jordan frame in $V^{+}$corresponding to $R^{+}$and $\mathfrak{a}$ is as in Part (i), then

$$
W \cap \mathfrak{a}=i L\left(\left\{\sum t_{i} e_{i} ; t_{i}<0\right\}\right)
$$

\section{Proof.}

(i) For any Euclidian Jordan algebra, $L(R)$ is a maximal abelian subspace in $L(V)$. Since by part (ii) of Theorem 1.6.1 $L\left(R^{+}\right)$is a maximial abelian subspace of $L\left(V^{+}\right)$, it follows that $i L\left(R^{+}\right)$is maximal abelian in $i L\left(V^{+}\right)=\mathfrak{q}_{\mathfrak{k}}(c f$. Eqn. (1.1)).

(ii) We have seen in Section 1.3 that $W \cap \mathfrak{q}_{\mathfrak{k}}=-i L\left(\Omega^{+}\right)$. Since $\Omega^{+}$is the cone of squares in $V^{+}$, we have $\Omega^{+} \cap R^{+}=\left\{\sum t_{i} e_{i} ; t_{i}>0\right\}$, and the claim follows directly from the definition of $\mathfrak{a}$ in part (i).

Proposition 1.6.5. - Assume that $X$ is irreducible. Then the following are equivalent: 
(i) $\alpha$ is non-split,

(ii) the rank of $X$ is equal to the split rank of $X$ (i.e. $X$ admits both compact and non-compact Cartan subspaces).

Proof. - If $\alpha$ is non-split, then $\mathfrak{a}_{1}:=\left\{v+j_{*} v ; v \in R^{-}\right\}$is a Cartan subspace contained in $\mathfrak{q}_{\mathfrak{p}}$ having the same dimension as $\mathfrak{a}$. If $\alpha$ is split, then one checks, using the classification, that the rank of $\mathfrak{q}_{\mathfrak{p}}$ is always strictly lower than the rank of $V^{+}$. (According to Remark 1.6.3, these are the cases I.2 and III.2 from Table 1.5.1; note that in case IV. $2 V^{+}$and $V^{-}$ have both rank equal to 2 , but this case is excluded since in case IV.2 $X$ is not simple. In Jordan theoretic terms, the rank of $\mathfrak{q}_{\mathfrak{p}}$ is equal to the rank of the Jordan triple system $V^{-}$whose isomorphism class is indicated in Table 1.5.1.)

\subsection{Peirce decomposition and root structure.}

Recall that the space $L(R)$ defined by a Jordan frame $c_{1}, \ldots, c_{r}$ is maximal abelian in $L(V)$. The weight decomposition of $V$ under the action of the abelian Lie algebra $L(R)$ is given by the Peirce decomposition (cf. [FK94, Th. IV.2.1])

$$
V=\bigoplus_{i=1}^{r} \mathbb{R} c_{i} \oplus \bigoplus_{i<j} V_{i j}
$$

where $V_{i j}$ for $i \neq j$ is the intersection of the $\frac{1}{2}$-eigenspaces of $L\left(c_{i}\right)$ and $L\left(c_{j}\right)$. In other words, if we denote by $c_{1}^{*}, \ldots, c_{r}^{*}$ the basis of $L(R)^{*}$ dual to $L\left(c_{1}\right), \ldots, L\left(c_{r}\right)$, then $V_{i j}$ is the weight space for the weight $\frac{1}{2}\left(c_{i}^{*}+c_{j}^{*}\right)$. For a simple algebra $V$, all the spaces $V_{i j}(i \neq j)$ have the same dimension called the genus of $V$ and denoted by $d$. We then have the relation

$$
n=r+\frac{1}{2} d r(r-1) \text {. }
$$

\subsubsection{Action of an involution on the Peirce spaces.}

Assume that $\alpha$ is an involution with $\alpha(R)=R$. We analyze the behavior of the spaces $V_{i j}$ under $\alpha$ in the cases corresponding to Remark 1.6.3:

- Split case. - It is immediate that all Peirce spaces $V_{i j}$ are stable under $\alpha$. We assume first that $V^{+}$is simple and consider the matrix cases (I.2, III.2, V.2). Then there is a structure of Euclidian Hurwitz algebra on $V_{i j}$ (see [FK94,Prop. V.3.4]), and $\alpha$ acts on $V_{i j}$ by a conjugation of $\mathbb{C}, \mathbb{H}$ or $\left(\mathbb{O}\right.$ according to the cases $d=2,4$ or 8 . The Peirce spaces for $V^{+}$are the $\alpha$-fixed parts of the Peirce spaces of $V$, having half the dimension of the latter. In the case of rank 2 there remains only Type IV.2 in which $X$ is not irreducible and which we will not consider.

TOME $126-1998-\mathrm{N}^{\circ} 3$ 
If $V^{+}$is not simple, then $\alpha=P(w)$ is a Peirce reflection ( $c f$. Remark 1.5.3) with $w=c_{1}+\ldots+c_{p}(1 \leq p<r)$, and then $\alpha$ acts trivially on $V_{i j}$ for $1 \leq i, j \leq p$ and for $p<i, j \leq r$ and by -1 on the other $V_{i j}$ 's.

- Non-split case. - The rank of $V$ is even, $r=2 r_{+}$, and we order the idempotents such that $c_{r_{+}+j}=\alpha\left(c_{j}\right)$. Then the idempotents $e_{j}$ of $V^{+}$are $e_{j}=c_{j}+\alpha c_{j}, j=1, \ldots, r_{+}$. In the matrix cases (I.3 and II.2) the Peirce-structure of the Euclidean Jordan algebra $V^{+}$w.r.t. the $e_{i}$ looks like the Peirce-structure of $V$, but with $d$ replaced by $d_{+}=2 d$. Let for $i, j=1, \ldots, r_{+}$

$$
U_{i j}:=V\left(L\left(e_{i}\right), \frac{1}{2}\right) \cap V\left(L\left(e_{j}\right), \frac{1}{2}\right)
$$

be the intersection of the $\frac{1}{2}$-eigenspaces of $L\left(e_{i}\right)$ and $L\left(e_{j}\right)$ in $V$. Then $U_{i j} \cap V^{+}$for $i<j$ are the Peirce-spaces of $V^{+}$. On the other hand, we get from the definition of the Peirce-spaces $V_{i j}$ of $V$ that

$$
U_{i j}=V_{i j} \oplus V_{i+r_{+}, j+r_{+}} \oplus\left(V_{i, j+r_{+}}+V_{j, i+r_{+}}\right),
$$

and thus $V=R \oplus \bigoplus_{i \leq j} U_{i j}$. Since we know already that $\operatorname{dim}\left(U_{i j} \cap V^{+}\right)=$ $d_{+}=2 d$, we conclude that the dimension of $U_{i j} \cap V^{-}$is $2 d$ if $i<j$ and $d$ if $i=j$. In case of rank 2 there remains only Case IV.3: there is just one Peirce-space in $V$; it belongs to $V^{-}$.

Finally, if $V$ is not simple, then we are in the Cayley-type case. Here $V^{+} \cong V^{-} \cong V_{1}$, and the decomposition of $V^{+}$and $V^{-}$w.r.t. the idempotents of $V^{+}$coincides with the usual Peirce-decomposition in $V_{1}$.

\subsubsection{Root structure.}

The complexification of the Lie algebra $\mathfrak{g}$ is given by

$$
\mathfrak{g}_{\mathbb{C}}=\mathfrak{c o}\left(V_{\mathbb{C}}\right)^{(-\alpha)_{*}}=V_{\mathbb{C}}^{-} \oplus \mathfrak{s t r}\left(V_{\mathbb{C}}\right)^{\alpha_{*}} \oplus j_{*} V_{\mathbb{C}}^{-} .
$$

Since $\mathfrak{a}=L\left(R^{+}\right)$is a compact Cartan subspace in $\mathfrak{q}$ (Prop. 1.6.4), the adjoint action of $\mathfrak{a}_{\mathbb{C}}$ on the Lie algebra $\mathfrak{g}_{\mathbb{C}}$ is diagonalizable. Let

$$
\Delta:=\Delta\left(\mathfrak{g}_{\mathbb{C}}, \mathfrak{a}_{\mathbb{C}}\right)
$$

be the root system of $\mathfrak{g}_{\mathbb{C}}$ with respect to $\mathfrak{a}_{\mathbb{C}}=L\left(R^{+}\right)_{\mathbb{C}}$. Since the action of $\mathfrak{a}_{\mathbb{C}}$ commutes with $-\mathrm{id}_{V_{\mathbb{C}}}$, the root spaces are contained in the homogeneous parts of the graded Lie algebra $\mathfrak{g}_{\mathbb{C}}$, and the root system $\Delta$ decomposes as

$$
\begin{aligned}
\Delta & =\Delta_{1} \cup \Delta_{0} \cup \Delta_{-1}, & \Delta_{1} & =\Delta\left(V_{\mathbb{C}}^{-}, \mathfrak{a}_{\mathbb{C}}\right), \\
\Delta_{0} & =\Delta\left(\mathfrak{s t r}\left(V_{\mathbb{C}}\right)^{\alpha_{*}}, \mathfrak{a}_{\mathbb{C}}\right), & \Delta_{-1} & =-\Delta_{1} .
\end{aligned}
$$

BULLETIN DE LA SOCIÉTÉ MATHÉMATIQUE DE FRANCE 
The roots from $\Delta_{0}$ are called compact and the roots from $\Delta_{1} \cup \Delta_{-1}$ noncompact. All roots are real-valued on the real form $\mathfrak{a}=L\left(R^{+}\right)$of $\mathfrak{a}_{\mathbb{C}} \cdot \mathrm{A}$ root is called positive if it is positive on the positive Weyl chamber

$$
\left\{\sum_{i} t_{i} e_{i} ; 0 \leq t_{1} \leq \ldots \leq t_{r}\right\} \text {. }
$$

As usual,

$$
\rho=\frac{1}{2} \sum_{\alpha \in \Delta^{+}} m_{\alpha} \alpha
$$

is the half-sum of the positive roots, weighted by the dimension $m_{\alpha}$ of the root spaces.

In Theorem 1.8.3 we will prove that, if $X$ is irreducible and not of type IV.3, then $\mathfrak{s t r}\left(V_{\mathbb{C}}\right)^{\alpha_{*}}$ and $\mathfrak{s t r}\left(V_{\mathbb{C}}^{+}\right)$are canonically isomorphic. This permits to describe $\Delta_{k}$, for $k=-1,0,1$, in terms of the Peirce decompositions of $V^{+}$and $V$. The details are given below. For simplicity of notation, we identify $\mathfrak{a}_{\mathbb{C}}$ with $R_{\mathbb{C}}^{+}$. Let $e_{1}, \ldots, e_{r_{+}}$be the Jordan frame belonging to $R^{+}$and $e_{1}^{*}, \ldots, e_{r_{+}}^{*}$ be its dual basis, considered as basis of $\mathfrak{a}_{\mathbb{C}}^{*}$. Let $V$ be of dimension $n$, rank $r$ and with genus $d$ and denote by a lower index + the corresponding constants for $V^{+}$.

- Case $A: X=G / H$ is simple and not of Type IV.3. - In this case $V^{+}$is a simple Jordan algebra, and Theorem 1.8.3 shows that $\mathfrak{s t r}(V)^{\alpha_{*}} \cong \mathfrak{s t r}\left(V^{+}\right)$. Thus, according to [FK94, p. 212],

$$
\begin{aligned}
& \Delta_{0}=\Delta\left(\mathfrak{s t r}\left(V_{\mathbb{C}}^{+}\right), \mathfrak{a}_{\mathbb{C}}\right)=\left\{\frac{1}{2}\left(e_{j}^{*}-e_{k}^{*}\right) ; j \neq k, j, k=1, \ldots, r_{+}\right\}, \\
& \rho_{0}=\rho\left(\Delta_{0}\right)=\frac{1}{4} d_{+} \sum_{j<k}\left(e_{k}^{*}-e_{j}^{*}\right)=\frac{1}{4} d_{+} \sum_{j}(2 j-r-1) e_{j}^{*} .
\end{aligned}
$$

The root system $\Delta_{1}:=\Delta\left(V^{-}, \mathfrak{a}\right)$ can be determined from the Peirce decompositions of $V$ and $V^{+}: V^{-}$is the sum of the weight spaces of a not appearing in $V^{+}$. According to Section 1.7 .1 we obtain:

(i) Case A.1: $\alpha$ split, i.e. $r_{+}=r$. - Note that for type IV such an involution does not exist (since we exclude case IV.2 in which $X$ is not irreducible). Thus we are in the matrix cases where $\alpha$ acts by a conjugation on the Euclidian Hurwitz algebra isomorphic to the $V_{i j}$ 's. Thus

$$
\Delta_{1}=\Delta\left(V_{\mathbb{C}}^{-}, \mathfrak{a}_{\mathbb{C}}\right)=\left\{\frac{1}{2}\left(e_{j}^{*}+e_{k}^{*}\right) ; j, k=1, \ldots, r_{+}, j<k\right\},
$$

and the corresponding root space dimensions are $d_{+}=\frac{1}{2} d$ with $d=2$ in Case I. $2, d=4$ in Case III. 2 and $d=8$ in Case V.2. Therefore

$$
\rho=\rho_{0}+\rho_{1}=\frac{1}{8} d\left(\sum_{j<k}\left(e_{k}^{*}-e_{j}^{*}\right)+\sum_{j<k}\left(e_{k}^{*}+e_{j}^{*}\right)\right)=\frac{1}{4} d \sum_{j}(j-1) e_{j}^{*} .
$$

TOME $126-1998-\mathrm{N}^{\circ} 3$ 
(ii) Case A.2: $\alpha$ non-split, i.e. $r_{+}=\frac{1}{2} r$. - There are $r_{+}$one dimensional weight spaces in $V^{-}$corresponding to $R^{-}$. In the matrix cases (I.3 with $d=2$, II.2 with $d=1$ ) there are in addition weight spaces for the weight $\frac{1}{2}\left(e_{j}^{*}+e_{k}^{*}\right)(j<k)$ with dimension $2 d$ and for the weight $e_{k}^{*}$ with dimension $d$. Thus

$$
\begin{aligned}
\Delta_{1} & =\left\{e_{j}^{*}, \frac{1}{2}\left(e_{j}^{*}+e_{k}^{*}\right) ; j, k=1, \ldots, r_{+}, j<k\right\}, \\
\rho & =\rho_{0}+\rho_{1}=\frac{1}{2} d \sum_{j<k}\left(e_{k}^{*}-e_{j}^{*}\right)+\frac{1}{2}(d+1) \sum_{j} e_{j}^{*}+\frac{1}{2} d \sum_{j<k}\left(e_{j}^{*}+e_{k}^{*}\right) \\
& =\frac{1}{2}(d+1) \sum_{j} e_{j}^{*}+d \sum_{j}(j-1) e_{j}^{*} .
\end{aligned}
$$

For the Cayley-type we have $d_{+}=d, n_{+}=\frac{1}{2} n$, and the decomposition of $V^{+} \cong V^{-}$with respect to $\mathfrak{a}$ is the usual Peirce decomposition of $V^{+}$ with $d_{+}=d$ :

$$
\begin{aligned}
\Delta_{1} & =\left\{e_{j}^{*}, \frac{1}{2}\left(e_{j}^{*}+e_{k}^{*}\right) ; j, k=1, \ldots, r_{+}, j<k\right\}, \\
\rho & =\frac{1}{2} \sum_{j} e_{j}^{*}+\frac{1}{4} d \sum_{k<j}\left(e_{k}^{*}-e_{j}^{*}+e_{k}^{*}+e_{j}^{*}\right)=\frac{1}{2} \sum_{j} e_{j}^{*}+\frac{1}{2} d \sum_{j}(j-1) e_{j}^{*} .
\end{aligned}
$$

Case B: Case IV.3 $(X \cong \mathrm{SO}(2, n-1) / \mathrm{SO}(1, n-1))$. - We have $r_{+}=\frac{1}{2} r=1$; thus $e_{1}=c_{1}+c_{2}$ is the unit element $e$ and $R^{+}=\mathbb{R} e$, $n_{+}=1$. Clearly $\Delta_{1}=\left\{e^{*}\right\}$ with weight space $V^{-}$. Since $L\left(R^{+}\right)=\mathbb{R} \operatorname{id}_{V}$, we have $\Delta_{0}=\Delta\left(\mathfrak{s t r}\left(V_{\mathbb{C}}\right)^{\alpha_{*}}, L\left(R^{+}\right)_{\mathbb{C}}\right)=\emptyset$, and thus

$$
\rho=\frac{1}{2}(n-1) e^{*} \text {. }
$$

Case C: $X$ is not irreducible. - If we discard the case IV.2, we may assume that $\alpha$ is a Peirce involution: $\alpha=P(w), w=c_{1}+\cdots+c_{p}, r_{1}=p$, $r_{-1}=q=r-p, d_{1}=d_{-1}=d, n_{1}=p+\frac{1}{2} d p(p-1), n_{-1}=q+\frac{1}{2} d q(q-1)$.

$$
\begin{aligned}
\Delta_{0} & =\Delta\left(\mathfrak{s t r}\left(V^{+}\right), \mathfrak{a}\right) \\
& =\left\{\frac{1}{2}\left(c_{j}^{*}-c_{k}^{*}\right) ; j \neq k, 1 \leq j, k \leq q \text { or } q<j, k \leq r\right\}, \\
\Delta_{1} & =\left\{\frac{1}{2}\left(e_{j}^{*}+e_{k}^{*}\right) ; 1 \leq j \leq p<k \leq r\right\}, \\
\rho & =\frac{1}{4} d \sum_{j=1}^{p}(2 j-p-1) e_{j}^{*}+\sum_{j=p+1}^{r}(2 j-q-1) e_{j+p}^{*}+\sum_{1 \leq j \leq p<k \leq r}\left(e_{j}^{*}+e_{k}^{*}\right) \\
& =\frac{1}{4} d \sum_{j=1}^{r}(2 j-2 p-r-1) e_{j}^{*} .
\end{aligned}
$$

BULLETIN DE LA SOCIÉTÉ MATHÉMATIQUE DE FRANCE 
1.8. The behavior of the structure group under involutions. In this subsection $\alpha$ is an involution of an arbitrary semisimple Jordan algebra $V$. According to [Sa80, I.7.3], the structure algebra $\mathfrak{s t r}\left(V^{-}\right)$of the non-degenerate JTS $V^{-}$is equal to the inner structure algebra

$$
V^{-} \square_{-} V^{-}
$$

generated by $v \square_{-} w\left(v, w \in V^{-}\right)$; here $v \square_{-} w$ is the restriction to $V^{-}$of

$$
v \square w=L(v w)+[L(v), L(w)] .
$$

Similarly,

$$
\mathfrak{s t r}\left(V^{+}\right)=V^{+} \square_{+} V^{+} .
$$

In this subsection we show (in a classification free way) that "generically" $\mathfrak{s t r}\left(V^{+}\right)$and $\mathfrak{s t r}\left(V^{-}\right)$can be identified. See [Hw69] for similar results.

Proposition 1.8.1. - Let $V$ be a semisimple Jordan algebra and $\alpha$ an involution of $V$. Then the following extension homomorphisms $i_{ \pm}$and restriction homomorphisms $r_{ \pm}$are well defined:

$$
\begin{gathered}
i_{ \pm}: \mathfrak{s t r}\left(V^{ \pm}\right)=V^{ \pm} \square_{ \pm} V^{ \pm} \longrightarrow \mathfrak{s t r}(V)^{\alpha_{*}}, \quad v \square_{ \pm} w \longmapsto v \square w \\
r_{ \pm}: \mathfrak{s t r}(V)^{\alpha_{*}} \longrightarrow \mathfrak{s t r}\left(V^{ \pm}\right), \quad X \longmapsto X_{\mid V^{ \pm}}
\end{gathered}
$$

They satisfy $r_{ \pm} \circ i_{ \pm}=\operatorname{id}_{\mathfrak{s t r}\left(V^{ \pm}\right)}$. In particular, $i_{ \pm}$is injective and $r_{ \pm}$is surjective.

Proof. - Let us show that the formula for $i_{ \pm}$indeed yields a well defined map. In fact, the inclusion $V^{ \pm} \subset V$ is a homomorphism of nondegenerate JTS, and by a result of Neher ( $c f$. [Sa80, p. 39/40]), such a homomorphism uniquely extends to a homomorphism of the associated graded Lie algebras which by restriction to $\mathfrak{s t r}\left(V^{ \pm}\right)$precisely yields $i_{ \pm}$. Now, if $v, w \in V^{ \pm}$,

$$
\alpha \circ(v \square w) \circ \alpha=(\alpha v) \square(\alpha w)=v \square w,
$$

and therefore the image of $i_{ \pm}$is contained in $\mathfrak{s t r}(V)^{\alpha_{*}}$.

We now show that the restriction maps are well defined: it is clear that $V^{+}$and $V^{-}$are stable under $\operatorname{Str}(V)^{\alpha_{*}}$ and under $\mathfrak{s t r}(V)^{\alpha_{*}}$. Further, the restriction of $X \in \mathfrak{s t r}(V)^{\alpha_{*}}$ to the subspaces $V^{ \pm}$belongs to the respective structure algebras: this follows easily from the decomposition

$$
\mathfrak{s t r}(V)^{\alpha_{*}}=\operatorname{Der}(V)^{\alpha_{*}} \oplus L\left(V^{+}\right) .
$$

Finally, it is clear that $r_{ \pm} \circ i_{ \pm}=\operatorname{id}_{\mathfrak{s t r}\left(V^{ \pm}\right)}$. $\square$

$$
\text { TOME } 126-1998-\mathrm{N}^{\circ} 3
$$


We say that $V^{+}$and $V^{-}$commute if

$$
\left[i_{-}\left(\mathfrak{s t r}\left(V^{-}\right)\right), i_{+}\left(\mathfrak{s t r}\left(V^{+}\right)\right)\right]=0
$$

Theorem 1.8.2. - Assume that $V^{-}$is simple and that $V^{+}$and $V^{-}$ don't commute.

(i) If $V^{+}$is simple, then $r_{-}$is injective and $i_{-}$is surjective.

(ii) If $V^{+}$is not simple, then $\alpha$ is a Peirce reflection $P(w)$ and $r_{-}$ has a one dimensional kernel given by $\mathbb{R} L(w)$; then $r_{+}(\mathbb{R} L(w))$ is in the center of $\mathfrak{s t r}\left(V^{+}\right)$.

(iii) $r_{+}$is injective and $i_{+}$is surjective.

Proof. - By Proposition 1.8.1, $i_{ \pm} \circ r_{ \pm}$is a projection onto the image of $i_{ \pm}$; therefore

$$
\mathfrak{s t r}(V)^{\alpha_{*}}=\operatorname{im} i_{+} \oplus \operatorname{ker} r_{+}=\operatorname{im} i_{-} \oplus \operatorname{ker} r_{-}
$$

We will now determine the kernel of the homomorphism

$$
r_{ \pm} \circ i_{\mp}: \mathfrak{s t r}\left(V^{\mp}\right) \longrightarrow \mathfrak{s t r}\left(V^{ \pm}\right), \quad v \square_{\mp} w \longmapsto(v \square w)_{\mid V^{ \pm}}
$$

Assume first that both $V^{-}$and $V^{+}$are simple. Then it is known that the center of the respective structure algebras is one dimensional, given by scalar multiples of the identity, and that their derived algebras are simple. Therefore $r_{+} \circ i_{-}$and $r_{-} \circ i_{+}$must be inverse isomorphisms of the derived algebras. In fact, if the intersection of the derived algebras with the respective kernels were not zero, $V^{+}$and $V^{-}$would commute. In particular, we see that $i_{\mp} \circ r_{\mp} \circ i_{ \pm}=i_{ \pm}$on the derived algebra.

We now prove that

$$
r_{ \pm} \circ i_{\mp}\left(\mathrm{id}_{V \mp}\right)=\mathrm{id}_{V^{ \pm}}
$$

First, $i_{+}\left(\operatorname{id}_{V^{+}}\right)=i_{+}\left(L(e)_{\mid V^{+}}\right)=\mathrm{id}_{V}$, therefore $r_{-} \circ i_{+}\left(\operatorname{id}_{V^{+}}\right)=\mathrm{id}_{V^{-}}$. According to $[\mathrm{Sa} 80, \mathrm{I} .6 .1]$, we can write

$$
\operatorname{id}_{V^{-}}=\sum_{i} c_{i} \square_{-} c_{i}^{\prime}
$$

where $\left(c_{i}\right)$ and $\left(c_{i}^{\prime}\right)$ are dual basis of $V^{-}$with respect to the trace form $(v, w) \mapsto \operatorname{Tr}\left(v \square_{-} w\right)$, and similarly for $V^{+}$with a basis $\left(d_{i}\right)$ of $V^{+}$. 
Because $V^{+}$and $V^{-}$are simple, the respective trace forms are multiples of the respective restrictions of the trace form of $V$. Hence

$$
\left(\frac{\operatorname{dim} V^{+}}{\operatorname{dim} V} d_{i}^{\prime}, \frac{\operatorname{dim} V^{-}}{\operatorname{dim} V} c_{j}^{\prime}\right)
$$

is the dual basis of the basis $\left(d_{i}, c_{j}\right)$ of $V$. One deduces that

$$
i_{+}\left(\mathrm{id}_{V^{+}}\right)+i_{-}\left(\mathrm{id}_{V^{-}}\right)=\sum_{i} c_{i} \square c_{i}^{\prime}+\sum_{i} d_{i} \square d_{i}^{\prime}=2 \mathrm{id}_{V}
$$

Since $i_{+}\left(\operatorname{id}_{V^{+}}\right)=\mathrm{id}_{V}$, we get $i_{-}\left(\operatorname{id}_{V^{-}}\right)=\mathrm{id}_{V}$. Thus $r_{ \pm} \circ i_{\mp}$ is an isomorphism on the center and, by what we have seen above, on all of $\operatorname{str}\left(V^{ \pm}\right)$.

We have proved that $i_{+}$and $i_{-}$have the same image. This implies that their complements are equal:

$$
\operatorname{ker} r_{+}=\operatorname{ker} r_{-} \text {. }
$$

But clearly ker $r_{+} \cap \operatorname{ker} r_{-}=0$, and hence $r_{ \pm}$and $i_{ \pm}$are isomorphisms.

It remains to consider the case when $V^{+}$is not simple. According to Remark 1.5.3, $\alpha$ is then a Peirce reflection $P(w)$. By standard facts on the Peirce decomposition ( $c f .\left[\right.$ FK94, p. 65]), $V^{+}$is then the 0-eigenspace of $L(w)$ and $V^{-}$is the direct sum of the +1-eigenspace $V_{1}$ and the -1eigenspace $V_{-1}$ of $L(w)$; both are ideals in $V^{+}$. We first prove that the image of $i_{-}$is contained in the image of $i_{+}$. In fact, since $V^{-}$is assumed to be simple, the arguments given above still go through. We conclude that ker $r_{+} \subset$ ker $r_{-}$, and hence ker $r_{+}=0$ since $\operatorname{ker} r_{-} \cap \operatorname{ker} r_{+}=0$. Thus $i_{+}\left(\mathfrak{s t r}\left(V^{+}\right)\right)=\mathfrak{s t r}(V)^{\alpha_{*}}$, and it is then immediately verified that ker $r_{-}=\mathbb{R} L(w)$.

Proposition 1.8.3. - If $V$ is Euclidian and the space $X$ associated to $\alpha$ is irreducible, then $r_{-}: \mathfrak{s t r}(V)^{\alpha_{*}} \rightarrow \mathfrak{s t r}\left(V^{-}\right)$is bijective.

Proof. - Using the classification of irreducible spaces from Table 1.5.1, we see that in all cases with the exception of IV.3 $V^{+}$and $V^{-}$don't commute, and in these cases Theorem 1.8.2 implies the claim. In the remaining case IV.3 we have $V^{+}=\mathbb{R}$ and thus $\mathfrak{s t r}\left(V^{+}\right) \cong \mathbb{R}$, and the claim can be proved by similar arguments as in the proof of Theorem 1.8.2.

REMARK 1.8.4. - In case IV.2 the statement of Theorem 1.8.2 is false: indeed, $V^{+}$and $V^{-}$do commute, and $\mathfrak{s t r}(V)^{\alpha_{*}}$ contains a direct product of the simple parts of the non-isomorphic structure groups of $V^{+}$and $V^{-}$.

TOME $126-1998-\mathrm{N}^{\circ} 3$ 
REMARK 1.8.5. - The surjective homomorphisms

$$
\rho_{ \pm}: G^{ \pm} \longrightarrow G\left(D^{ \pm}\right)
$$

(cf. Section 1.2) have discrete kernel if and only if

$$
r_{ \pm}: \mathfrak{s t r}(V)^{\alpha_{*}} \longrightarrow \mathfrak{s t r}\left(V^{ \pm}\right)
$$

are bijective. In fact, the kernel of the derived homomorphism of Lie algebras $\dot{\rho}_{ \pm}: \mathfrak{g}_{\mathbb{C}}^{ \pm} \rightarrow \mathfrak{g}\left(D^{ \pm}\right)_{\mathbb{C}}$ is contained in the homogeneous part of degree 1 in $\mathfrak{g}_{\mathbb{C}}^{ \pm}$which is just $\mathfrak{s t r}\left(V_{\mathbb{C}}\right)^{\alpha_{*}}$. Thus Theorem 1.8.1 yields a criterion when $\rho_{ \pm}$is essentially injective.

\section{The spectrum of a family of weighted Bergman spaces}

In this section we fix an involution $\alpha$, the corresponding domain $\Xi \subset D$ and the compactly causal space $X=G / H$.

\subsection{The spaces $B_{\lambda, \mu}^{2}(\Xi)$ (proof of Theorem 1).}

We define the weighted Bergman spaces $B_{\mu}^{2}(D)$ and $B_{\lambda, \mu}^{2}(\Xi)$ by Equations (0.1) and (0.5).

Proposition 2.1.1. - A function $f \in B_{\mu, 0}^{2}(\Xi)$ extends to a holomorphic function on $D$.

Proof. - This follows from [Pe96, Th. 1.2] since $D \backslash \Xi$ is an analytic (even algebraic) set in $D$.

The map assigning to $f \in B_{\mu, 0}^{2}(\Xi)$ its holomorphic extension onto $D$ is a unitary bijection of $B_{\mu, 0}^{2}(\Xi)$ onto $B_{\mu}^{2}(D)$ which we will consider as an identification.

Corollary 2.1.2. - If $B_{\mu, 0}^{2}(\Xi)$ is not reduced to zero, then its reproducing kernel is given by

$$
K^{(\mu, 0)}(z, w)=(\operatorname{Det} B(z, \bar{w}))^{-\mu}
$$

with $B$ given by Equation (0.2).

Proof. - The preceding proposition implies that $B_{\mu, 0}^{2}(\Xi)$ and $B_{\mu}^{2}(D)$ have the same reproducing kernel. Now the claim follows from [FK94, Prop. XIII.1.4].

Proposition 2.1.3. - For all admissible parameters $k$,

$$
\mathcal{A}_{\mu, \lambda, 2 k}: B_{\mu, \lambda}^{2}(\Xi) \longrightarrow B_{\mu, \lambda+2 k}^{2}(\Xi), \quad f \longmapsto\left(f_{\alpha}\right)^{k} \cdot f
$$

is an isometry onto.

Proof. - This is immediate from the definition of the spaces $B_{\mu, \lambda}^{2}$ (Equation (0.5)). 
Corollary 2.1.4. - If $B_{\mu, 0}^{2}(\Xi)$ is not reduced to zero and $k$ is an admissible parameter, then the reproducing kernel of $B_{\mu, 2 k}^{2}(\Xi)$ is given by

$$
K^{(\mu, 2 k)}(z, w)=f_{\alpha}(z)^{k}(\operatorname{Det} B(z, \bar{w}))^{-\mu} \overline{f_{\alpha}(w)}{ }^{k} .
$$

Proof.-We drop the index $\mu$ and denote by $K^{(\lambda)}(z, w)$ the reproducing kernel corresponding to $\lambda$. Then

$$
\begin{gathered}
f(z)=\left(f \mid K_{z}^{(0)}\right)_{0}=\left(f_{\alpha}^{k} f \mid f_{\alpha}^{k} K_{z}^{(0)}\right)_{2 k}, \\
\left.\left(f_{\alpha}^{k} f\right)(z)=\left(f_{\alpha}^{k} f \mid f_{\alpha}^{k} K_{z}^{(0)} \overline{f_{\alpha}(z)}\right)^{k}\right)_{2 k} .
\end{gathered}
$$

Therefore

$$
K^{(2 k)}(z, w)=f_{\alpha}(z)^{k} K^{(0)}(z, w){\overline{f_{\alpha}(w)}}^{k}
$$

with $K^{(0)}$ from Corollary 2.1.2.

This completes the proof of Theorem 1. It is immediate from the definition of a positive kernel (cf. [FK94, p. 171]) that, for $k$ admissible, $K^{(\mu, 2 k)}$ is positive if and only if $K^{(\mu, 0)}$ is. This in turn is the case if and only if $\mu$ belongs to the Wallach set $\mathcal{W}$ given by Equation (0.9) (see [FK94, Th. XIII.2.7]). We will denote by $B_{\mu, 2 k}(\Xi)$ the corresponding "abstract" reproducing kernel space. Then

$$
\mathcal{A}_{\mu, 2 k}: B_{\mu, 0}(\Xi) \longrightarrow B_{\mu, 2 k}(\Xi)
$$

as in Proposition 2.1.3 is an isomorphism of reproducing kernel spaces.

\subsection{Group and semigroup actions.}

The following lemma describes how the group of holomorphic automorphisms of a circled complex domain acts on spaces of holomorphic functions.

Lemma 2.2.1. - Let $M=G(M) / U \subset E$ be a circled bounded symmetric domain in a finite dimensional complex vector space $E$, where $G(M)=\operatorname{Aut}(M)_{o}$ und $U$ is the stabilizer of the base point $0_{E}$. If $\left(\rho, \mathcal{V}_{\rho}\right)$ is a finite dimensional representation of $U_{\mathbb{C}}$, then the formula

$$
\left(\pi_{\rho}(g) f\right)(z):=\rho\left(\mathrm{d} g^{-1}(z)\right)^{-1} \cdot f\left(g^{-1} z\right)
$$

defines a linear action of $G(M)$ on the space $\mathcal{O}\left(M, \mathcal{V}_{\rho}\right)$ of holomorphic $\mathcal{V}_{\rho}$-valued functions on $M$. The same formula defines a linear action of the semigroup $S(M)^{-1}$, where

$$
S(M):=\left\{g \in G(M)_{\mathbb{C}} ; g(M) \subset M\right\} .
$$

TOME $126-1998-\mathrm{N}^{\circ} 3$ 
Proof. - It is known that, whenever $\mathrm{d} g^{-1}(z)$ exists for $g \in G(M)_{\mathbb{C}}$ and $z \in M$, it is an element of $U_{\mathbb{C}}(c f$. [Lo77, 8.15] or [Sa80, Lemma II.5.3]). Thus the term on the right hand side is well defined. Using the chain rule one easily verifies that $\pi_{\rho}(g h)=\pi_{\rho}(g) \pi_{\rho}(h)$.

REMARK 2.2.2. - The representation $\pi_{\rho}$ is equivalent to the subspace of the induced representation $\operatorname{Ind}_{U}^{G(M)} \rho$ given by holomorphic sections of the associated bundle over $G(M) / U=M$ with fiber $\mathcal{V}_{\rho}$ (cf. [FO95, p. 25]).

REMARK 2.2.3. - We want to apply the preceding lemma to the representation $\rho_{q}(u)=(\operatorname{Det} u)^{q}$ for $q$ a rational number, the case $q=\frac{1}{2}$ being of particular interest. If $V$ is a simple Jordan algebra, then $\rho_{q}$ is a well defined representation of $U$ if and only if $q$ is a multiple of $\frac{r}{n}$. (In fact, the equation $\Delta(g z)=\operatorname{Det}(g)^{r / n} \Delta(z)$ for all $z \in V_{\mathbb{C}}, g \in \operatorname{Str}\left(V_{\mathbb{C}}\right)$ shows that $g \mapsto \operatorname{Det}(g)^{r / n}$ is a well defined character of $\operatorname{Str}\left(V_{\mathbb{C}}\right)$. The converse is deduced from the fact that every relative invariant, i.e. a function on $\Omega$ transforming according to a character, is a power of the Jordan determinant $\Delta ; c f$. Remark 1.5.5.) In order to be able to apply a version of Lemma 2.2.1, we construct explicitly an $m$-fold covering group of $G(M)$ ( $c f$. [KØ97, Section 5, "Principle 1"] for more details).

Since $M$ is simply connected, for any $g \in G(M)$ the nowhere vanishing polynomial $\chi_{g}(z)=\operatorname{det}\left(\mathrm{d} g^{-1}(z)\right)^{-1}$ has $m$ holomorphic $m$-th roots. Thus the first projection of

$$
\widetilde{G}(M)_{m}:=\left\{(g, F) \in G(M) \times \mathcal{O}(M) ; \forall z \in M: F(z)^{m}=\chi_{g}(z)\right\}
$$

defines an $m$-fold covering

$$
p_{m}: \widetilde{G}(M)_{m} \longrightarrow G(M) .
$$

If, in this definition, we replace the term $F(z)^{m}$ by $\mathrm{e}^{F(z)}$, then we obtain a covering

$$
p_{\infty}: \widetilde{G}(M)_{\infty} \longrightarrow G(M)
$$

of infinite order. We assume from now on that $m \in \mathbb{N} \cup\{\infty\}$. The following product defines a group structure on $\widetilde{G}(M)_{m}$ :

$$
(g, F) \cdot\left(g^{\prime}, F^{\prime}\right)=\left(g g^{\prime}, F^{\prime \prime}\right), \quad F^{\prime \prime}(z)=F\left(g^{\prime} z\right) F^{\prime}(z) .
$$

Since a root of $\chi_{g}$ is determined by its value at the base point $0, \widetilde{G}(M)_{m}$ may topologically also be described as

$$
\widetilde{G}(M)_{m}=\left\{(g, z) \in G(M) \times \mathbb{C} ; z^{m}=\chi_{g}(0)\right\} .
$$


If $\widetilde{U}_{m}=p_{m}^{-1}(U)$, then the second projection $\widetilde{U}_{m} \rightarrow \mathbb{C},(u, z) \mapsto z$ is a character whose $m$-th power is the character Det; therefore we denote it by $\operatorname{Det}^{1 / m}$.

All definitions go through for $G(M)$ replaced by the semigroup $S(M)$; we thus define $m$-th order covering semigroups $\widetilde{S}(M)_{m}$.

Now we return to the setup from Section 2.1 with $M:=D$. Note that $G \subset G(D)$ and $G \exp (i W) \subset S(D)$, where $W \subset \mathfrak{q}$ is the cone defined in Section 1.3. Note that

$$
s=g \exp (i X) \longmapsto s^{*}=\exp (i X) g^{-1}
$$

defines an anti-holomorphic involution on $S(D)$ and similarly for the covering semigroups. Recall the concept of a Hermitian representation of an involutive semigroup $S$ on a pre-Hilbert space $\mathfrak{H}^{0}$ from [Ne98, Def. II.3.3]: The vector space $B_{0}\left(\mathfrak{H}^{0}\right)$ of linear operators $A: \mathfrak{H}^{0} \rightarrow \mathfrak{H}^{0}$ for which a formal adjoint exists is an involutive semigroup, and a Hermitian representation of $S$ on $\mathfrak{H}^{0}$ is a semigroup homomorphism $\pi: S \rightarrow B_{0}\left(\mathfrak{H}^{0}\right)$ preserving the involutions, i.e. $\pi\left(s^{*}\right)=\pi(s)^{*}$.

Proposition 2.2.4. - The formula

$$
\left(\pi_{\mu}(g) f\right)(z):=\left(\operatorname{Det} \mathrm{d} g^{-1}(z)\right)^{\mu} f\left(g^{-1} z\right)
$$

defines a unitary representation of $G(D)_{\infty}$ and a Hermitian holomorphic contractive representation of the semigroup $S(D)_{\infty}^{-1}$ in $B_{\mu}(D)$.

Proof.-Because of the construction of the covering (semi-)group and of Lemma 2.2.1, $\pi_{\mu}$ is an action on the space of holomorphic functions on $D$. We have to show that it preserves (resp. contracts) the norm of $B_{\mu}(D)$.

The relation

$$
B(g \cdot z, \overline{g \cdot w})=\mathrm{d} g(z) B(z, \bar{w})(\mathrm{d} g(w))^{*}
$$

for $g \in G(D)(c f .[\mathrm{Be} 98,1.3 .(9)]$ or $[\mathrm{Sa} 80, \mathrm{p} .65])$ yields

$$
K_{\mu}(g \cdot z, g \cdot w)=(\operatorname{Det} \mathrm{d} g(z))^{-\mu} K_{\mu}(z, w) \overline{\operatorname{Det}(\mathrm{d} g(w))}^{-\mu}
$$

which is equivalent to

$$
\pi_{\mu}(g) \cdot K_{w}=\overline{\operatorname{Det}(\mathrm{d} g(w))}^{-\mu} K_{g \cdot w}
$$

TOME $126-1998-\mathrm{N}^{\circ} 3$ 
Since $B_{\mu}(D)$ is spanned by the $K_{w}$, the space is stable under $\pi_{\mu}$. Further, (2.1) and (2.2) together imply that

$$
\left(\pi_{\mu}(g) K_{w} \mid \pi_{\mu}(g) K_{z}\right)_{\mu}=K(z, w)=\left(K_{w} \mid K_{z}\right)
$$

and therefore $\pi_{\mu}$ is a unitary representation of $G(D)_{\infty}$.

Analytic extension of (2.1) in the $g$-variable yields

$$
\left(\operatorname{Det} \mathrm{d} s(z)^{-1}\right)^{-\mu} K_{\mu}(s \cdot z, w)=K_{\mu}\left(z, s^{*} \cdot w\right){\overline{\operatorname{Det}\left(\mathrm{d} s^{*}(w)^{-1}\right)^{-\mu}}}^{-\mu}
$$

But then [Ne98, Prop. II.4.3] implies that $S(D)^{-1}$ acts on $B_{\mu}(D)$ via a Hermitian representation and the action of $S(D)_{\infty}^{-1}$ clearly is holomorphic.

It remains to show that any $g \in S(D)_{\infty}^{-1}$ acts by a contraction. We prove this first for $\mu=1$ : then $B_{\mu}=B_{\mu}^{2}$ is the classical Bergman space, and a change of variables yields immediately

$$
\left\|\pi_{1}(g) f\right\|_{1}^{2}=\int_{g^{-1} D}|f(z)|^{2} \mathrm{~d} z \leq \int_{D}|f(z)|^{2} \mathrm{~d} z=\|f\|_{1}^{2}
$$

since $g^{-1} D \subset D$. From this we deduce the result for general $\mu$ using Th. II.4.4 of [Ne98] which states that, in the situation we consider, for all $g \in S(D)_{\infty}^{-1}$,

$$
\left\|\pi_{\mu}(g)\right\|^{2}=\sup \left\{\left|\operatorname{Det} g^{-1}(z)\right|^{2 \mu} \frac{K_{\mu}(g z, g z)}{K_{\mu}(z, z)} ; z \in D, K_{\mu}(z, z)>0\right\} .
$$

In fact, we have just seen that for $\mu=1$ the right hand side is bounded by 1 , and since $K_{\mu}=K_{1}^{\mu}$, it is bounded by 1 for all positive $\mu$.

Note that if $\mu$ is rational, the previous proposition holds with $G(D)_{\infty}$ replaced by some $G(D)_{m}$ with finite $m$, and if $\mu=\frac{1}{2}$ one may choose $m=4$. In the following we assume that $m \in \mathbb{N} \cup\{\infty\}$ is suitably chosen. Now let $k$ be an admissible parameter ( $c f$. Section 2.1). Then we can push forward the unitary action of $G(D)_{m}$ by the isomorphism $\mathcal{A}_{\mu, 0,2 k}$ and obtain a unitary action $\pi_{\mu, 2 k}$ of $G(D)_{m}$ on $B_{\mu, 2 k}(\Xi)$ by

$$
\left(\pi_{\mu, 2 k}(g) f\right)(z)=f_{\alpha}(z)^{k}\left(\operatorname{Det} \mathrm{d} g^{-1}(z)\right)^{\mu}\left(f_{\alpha}^{-k} \cdot f\right)\left(g^{-1} z\right) .
$$

The same formula describes the pushforward of the holomorphic contractive action of the semigroup $S(D)_{m}^{-1}$. Let $G_{m} \subset G(D)_{m}$ be the preimage of $G \subset G(D)$ under the canonical projection $G(D)_{m} \rightarrow G(D)$ and $S_{m} \subset S(D)_{m}$ the preimage of $S:=G_{\mathbb{C}} \cap S(D) \subset S(D)$. 
Proposition 2.2.5. - Let $k$ be an admissible parameter.

(i) For all $g \in G_{m}$,

$$
\left(\pi_{\mu, 2 k}(g) f\right)(z)=\left(\operatorname{Det} \mathrm{d} g^{-1}(z)\right)^{\mu-2 k} f\left(g^{-1} z\right) .
$$

In particular,

$$
\left(\pi_{2 k, 2 k}(g) f\right)(z)=f\left(g^{-1} z\right) .
$$

By holomorphic extension, the same formulas hold for $g \in S_{m}$.

(ii) The kernel $K^{(2 k, 2 k)}$ is a $G_{m}$-invariant function: for all $g \in G_{m}$ and $z, w \in D$,

$$
K^{(2 k, 2 k)}(g z, g w)=K^{(2 k, 2 k)}(z, w)
$$

Proof.

(i) We prove first the following transformation law: for all $g \in G$ and $x, y$ where the following expressions are defined, we have

$$
P(g(x)+\alpha g(y))=\mathrm{d} g(x) P(x+\alpha y) j \alpha \mathrm{d} g(y)^{-1} j \alpha .
$$

In fact, this follows from the corresponding transformation property of $B_{\alpha}$ (see $[\mathrm{Be} 98,1.3 .(9)]$ ) by a calculation similar to [Be98, 2.1.2]. We observe further that $j \alpha \mathrm{d} g(y)^{-1} j \alpha$ is just the transposed of $\mathrm{d} g(y)$ with respect to the form $\operatorname{Tr} L(x \alpha y)$ ( $c f$. [Be98]) and therefore has the same determinant as $\mathrm{d} g(y)$; we deduce that for $g \in G_{m}$,

$$
f_{\alpha}\left(g^{-1} z\right)=\left(\operatorname{Det} \mathrm{d} g^{-1}(z)\right)^{2} f_{\alpha}(z)
$$

Now the claim follows using this transformation law in the expression for $\pi_{\mu, 2 k}$.

(ii) This can be verified directly from the transformation properties of $f_{\alpha}$ just proved, or it can be seen as an easy consequence of (i).

\subsection{The differential restriction operators.}

If $\alpha=\operatorname{id}_{V}$, then $G$ is the compact linear group $U$, and the decomposition of $B_{\mu}(D)$ with respect to this group is described in [FK94, Th. XIII.2.7]. We want to obtain a similar decomposition of this space under the action of $G$ (resp. $G_{m}$ ) for general $\alpha$. Recall that

$$
D^{-}=D \cap V_{\mathbb{C}}^{-}
$$

is a bounded symmetric domain on which $G$ acts transitively, and $D^{-}=G / K$ (Section 1.2). The basic idea, due to Jakobsen and Vergne

$$
\text { TOME } 126-1998-\mathrm{N}^{\circ} 3
$$


(see [JV79]), is to decompose $B_{\mu}(D)$ as a $G$-module by restricting functions and their transversal derivatives to $D^{-}$. For a smooth function $f: E \supset Y \rightarrow F$ defined on a domain $Y$ in a vector space $E$ with values in a vector space $F$, we denote by

$$
\mathrm{d} f: Y \longrightarrow \operatorname{Hom}(E, F)
$$

its ordinary total differential, and by

$$
\mathrm{d}^{k} f: Y \longrightarrow \operatorname{Hom}\left(S^{k} E, F\right)
$$

its $k$-th total differential; here $S^{k} E$ is the $k$-th symmetric power of $E$. If furthermore $f$ is holomorphic and scalar-valued, then $\mathrm{d}^{k} f$ is a holomorphic function with values in $\left(S^{k} E\right)^{*}$; we write $\mathrm{d}^{k} f \in \mathcal{O}\left(Y,\left(S^{k} E\right)^{*}\right)$.

Let $f$ be a holomorphic function $D \rightarrow F$. The restriction of the linear function

$$
\mathrm{d}^{k} f(z): S^{k} V_{\mathbb{C}} \longrightarrow F
$$

to the subspace $S^{k} V_{\mathbb{C}}^{+}$will be called the $V^{+}$-transversal $k$-th derivative of $f$ at $z$ and is denoted by $\mathrm{d}_{+}^{k} f(z)$. We now define the restriction operators

$$
\begin{array}{ll}
\mathcal{R}^{(0)}: \mathcal{O}(D, F) \longrightarrow \mathcal{O}\left(D^{-}, F\right), & f \longmapsto f_{\mid D^{-}} \\
\mathcal{R}^{(1)}: \operatorname{ker}\left(\mathcal{R}^{(0)}\right) \longrightarrow \mathcal{O}\left(D^{-}, \operatorname{Hom}\left(V_{\mathbb{C}}^{+}, F\right)\right), & f \longmapsto \mathrm{d}_{+} f_{\mid D^{-}}, \\
\quad \vdots & \\
\mathcal{R}^{(k)}: \operatorname{ker}\left(\mathcal{R}^{(k-1)}\right) \longrightarrow \mathcal{O}\left(D^{-}, \operatorname{Hom}\left(S^{k} V_{\mathbb{C}}^{+}, F\right)\right), & f \longmapsto \mathrm{d}_{+}^{k} f_{\mid D^{-}} .
\end{array}
$$

Proposition 2.3.1. - If $F$ is a $K_{m}$-module, then the maps $\mathcal{R}^{(k)}: \mathcal{O}(D, F) \supset \operatorname{ker}\left(\mathcal{R}^{(k-1)}\right) \rightarrow \mathcal{O}\left(D^{-}, \operatorname{Hom}\left(S^{k} V_{\mathbb{C}}^{+}, F\right)\right), \quad f \mapsto \mathrm{d}_{+}^{k} f_{\mid D^{-}}$ are $G_{m}$-equivariant maps with respect to the actions of $G_{m}$ defined by Lemma 2.2.1.

Proof. - The claim is verified by a direct computation: for $k=0$ it is immediate. For $k=1$ we differentiate

$$
\begin{aligned}
\mathrm{d}(g \cdot f)(z)= & \mathrm{d}\left(\rho\left(\mathrm{d} g^{-1}(\cdot)^{-1}\right) \cdot\left(f \circ g^{-1}\right)\right)(z) \\
= & \mathrm{d}\left(\rho\left(\mathrm{d} g^{-1}(\cdot)^{-1}\right)\right)(z) \cdot f\left(g^{-1}(z)\right) \\
& \quad+\rho\left(\mathrm{d} g^{-1}(z)\right)^{-1} \cdot\left(\mathrm{d} f\left(g^{-1}(z)\right) \circ \mathrm{d} g^{-1}(z)\right) .
\end{aligned}
$$

We evaluate for $f \in \operatorname{ker} \mathcal{R}^{(0)}$ and $z \in D^{-}$: then $f\left(g^{-1}(z)\right)=0$ since $g$ preserves $D^{-}$; therefore the first term vanishes, and the remaining term 
(considered as operator on $V_{\mathbb{C}}^{+}$) is just $(g \cdot \mathrm{d} f)(z)$. For $k>1$ we differentiate the above expression $k-1$ times, using chain and Leibnitz rule, and obtain a sum of multilinear terms containing differentials of $f$ of order $0,1, \ldots, k$. The terms containing differentials of $f$ of order less than $k$ vanish when we evaluate for $f \in \operatorname{ker} \mathcal{R}^{(k-1)}$ and $z \in D^{-}$, and only one term remains, namely

$$
\rho\left(\mathrm{d} g^{-1}(z)\right)^{-1} \cdot\left(\mathrm{d}^{k} f\left(g^{-1}(z)\right) \circ\left(S^{k} \mathrm{~d} g^{-1}(z)\right)\right),
$$

and this is precisely $\left(g \cdot \mathrm{d}^{k} f\right)(z)$ (when restricted to $S^{k} V_{\mathbb{C}}^{+}$).

Now let $\mathcal{H} \subset \mathcal{O}(D, F)$ be a $G$-invariant Hilbert subspace (i.e. a subspace with a structure of Hilbert space for which the point evaluations are continuous und such that $G$ acts unitarily in $\mathcal{H}$ ). Let $\mathcal{R}_{\mathcal{H}}^{(k)}$ be the restriction of $\mathcal{R}^{(k)}$ to $\mathcal{H}$.

Lemma 2.3.2. - For all $k \geq 0$, the kernels $\operatorname{ker} \mathcal{R}_{\mathcal{H}}^{(k)}$ are closed subspaces of $\mathcal{H}$, and the restriction operators $\mathcal{R}_{\mathcal{H}}^{(k)}$ are continuous.

Proof. - It follows from the Cauchy inequalities (cf. [Chab90, p. 30]) that the maps

$$
\mathrm{d}^{k}: \mathcal{O}(D, F) \longrightarrow \mathcal{O}\left(D, \operatorname{Hom}\left(S^{k} V_{\mathbb{C}}, F\right)\right), \quad f \longmapsto \mathrm{d}^{k} f
$$

are continuous with respect to the topologies of uniform convergence on compact sets. Therefore these maps and hence the differential restriction operators are continuous on $\mathcal{H}$, and their kernels are closed.

For $k>1$ we let $\mathcal{H}_{k}$ be the orthocomplement of $\operatorname{ker} \mathcal{R}_{\mathcal{H}}^{(k)}$ in $\operatorname{ker} \mathcal{R}_{\mathcal{H}}^{(k-1)}$, and let $\mathcal{H}_{0}$ be the orthocomplement of ker $\mathcal{R}_{\mathcal{H}}^{(0)}$ in $\mathcal{H}$. Since $G$ acts unitarily, it follows from Proposition 2.3.1 that $\mathcal{H}_{k}$ is a $G$-submodule of $\mathcal{H}$. Therefore

$$
\mathcal{R}_{\mathcal{H}}^{(k)}: \mathcal{H}_{k} \longrightarrow \mathcal{O}\left(D^{-}, \operatorname{Hom}\left(S^{k} V_{\mathbb{C}}^{+}, F\right)\right)
$$

is a $G$-isomorphism onto the image of $\mathcal{R}_{\mathcal{H}}^{(k)}$.

Proposition 2.3.3. - We have the following orthogonal G-invariant decomposition:

$$
\mathcal{H}=\bigoplus_{k=0}^{\infty} \mathcal{H}_{k}
$$

Proof. - It only remains to show that the sum $\bigoplus_{k} \mathcal{H}_{k}$ is dense in $\mathcal{H}$ : in fact, if $f \in\left(\bigoplus_{k} \mathcal{H}_{k}\right)^{\perp}$, then $f_{\mid D^{-}}=0$ since $f \in \mathcal{H}_{0}^{\perp}=\operatorname{ker} \mathcal{R}_{\mu}^{(0)}$. Further, all partial derivatives of $f$ in $V_{\mathbb{C}}^{+}$-direction vanish on $D^{-}$, and therefore the Taylor series of $f$ at any point of $D^{-}$is zero, implying that $f=0$ by analyticity.

TOME $126-1998-\mathrm{N}^{\circ} 3$ 
We transport the Hilbert structure of $\mathcal{H}_{k}$ via the isomorphism $\mathcal{R}_{\mathcal{H}}^{(k)}$ onto its image. This defines a Hilbert subspace (denoted by $\mathcal{R H}_{k}$ ) of $\mathcal{O}\left(D^{-}, \operatorname{Hom}\left(S^{k} V_{\mathbb{C}}^{+}, F\right)\right)$; in fact, the point evaluations of this space are continuous since they are given by differentials on $D$ which have the continuity property described in Lemma 2.3.2.

Since $G$ acts transitively on $D^{-}$, we can apply a theorem due to S. Kobayashi ( $c f$. [Ko68] for the square integrable version and [BH97, Th. 2.5] for the abstract kernel version) in order to decompose the space $\mathcal{R} \mathcal{H}_{k}$ into irreducible submodules. In our situation the theorem implies: $a$ $G$-invariant Hilbert subspace $\mathfrak{H}$ of $\mathcal{O}\left(D^{-}, E\right)$ is irreducible under $G$ if and only if $\mathfrak{H}(0):=\{f(0) \mid f \in \mathfrak{H}\}$ is irreducible under $K=G \cap U$, where $U$ is the maximal compact subgroup of $G(D)$ defined in Section 1.1. Put in another way: given an irreducible $K$-module $E_{0}$, there is at most one $G$ invariant Hilbert subspace $\mathfrak{H} \subset \mathcal{O}\left(D^{-}, U\right)$ with $\mathfrak{H}(0)=E_{0}$. If it exists, we denote it by $\mathcal{H}\left(D^{-}, E_{0}\right)$; by Kobayashi's theorem, it is irreducible. (Using the mean value property of holomorphic functions, one can show that $\mathcal{H}\left(D^{-}, E_{0}\right)$ is the $G$-module generated by all constant functions $D^{-} \rightarrow E_{0}$; cf. [Ach96, Section 2].)

Theorem 2.3.4. - Let $\mathcal{H} \subset \mathcal{O}(D, F)$ be a $G$-invariant Hilbert subspace. For $k=0,1,2, \ldots$ let

$$
\mathcal{R H}_{k}(0)=\bigoplus_{i} W_{k, i}
$$

be the decomposition into irreducible $K$-modules. Then $\mathcal{H}\left(D^{-}, W_{k, i}\right)$ is irreducible, and

$$
\mathcal{H} \cong \bigoplus_{i, k} \mathcal{H}\left(D^{-}, W_{k, i}\right)
$$

is the decomposition into irreducible G-modules.

Proof. - Since by Proposition 2.3.3 $\mathcal{H} \cong \bigoplus_{k} \mathcal{R H}_{k}$, we have to decompose $\mathcal{R} \mathcal{H}_{k}$ into irreducible $G$-modules. If $\mathcal{R H}_{k}=\bigoplus_{j} E_{j}$ is this decomposition, then one direction of Kobayashi's theorem tells us that $\mathcal{R H}_{k}(0)=\bigoplus_{j} E_{j}(0)$ is a decomposition into irreducible $K$-modules, and by the unicity statement we have $E_{j}=\mathcal{H}\left(D^{-}, E_{j}(0)\right)$. This implies the claim.

In the case where $F$ is one dimensional and $\mathcal{H}=B_{\mu}(D)$, we have the following, more precise, result:

Theorem 2.3.5. - Let $\mathbb{C}_{\mu}$ be the space $\mathbb{C}$ with the action of $K_{\infty}$ by $(\text { Det } k)^{-\mu}$, let $\operatorname{Pol}\left(V_{\mathbb{C}}^{+}, \mathbb{C}_{\mu}\right)$ be the space of holomorphic polynomials on 
$V_{\mathbb{C}}^{+}$with the action of $K_{\infty}$ by $k \cdot p=(\operatorname{Det} k)^{-\mu} \cdot\left(p \circ k^{-1}\right)$, and let

$$
\operatorname{Pol}\left(V_{\mathbb{C}}^{+}, \mathbb{C}_{\mu}\right)=\bigoplus_{j \in J} W_{j}
$$

be its decomposition into irreducible $K_{\infty}$-modules. Then for $\mu>\frac{1}{2}-\frac{r}{2 n}$

$$
B_{\mu}(D)=\bigoplus_{j \in J} \mathcal{H}\left(D^{-}, W_{j}\right)
$$

is the decomposition into irreducible G-modules.

Proof. - We apply the preceding theorem for $F=\mathbb{C}_{\mu}$ and $\mathcal{H}=B_{\mu}(D)$. By [FK94, Th. XIII.2.7], $B_{\mu}(D)\left(\mu>\frac{1}{2}-\frac{r}{2 n}\right)$ contains all holomorphic polynomials; one deduces that

$$
\mathcal{R} \mathcal{H}_{k}(0)=\operatorname{Hom}\left(S^{k} V_{\mathbb{C}}^{+}, \mathbb{C}_{\mu}\right)
$$

In fact, if $q: D^{-} \rightarrow\left(S^{k} V_{\mathbb{C}}^{+}\right)^{*}, z \mapsto q_{0}$ is a constant function, then one may find a holomorphic polynomial $p$ such that $\mathrm{d}_{+}^{k} p(z)=q(z)=q_{0}$ and $\mathrm{d}_{+}^{k-1} p(z)=0$ for all $z \in D^{-}$, namely $p(z)=(1 / k !) q_{0}\left(\frac{1}{2}(z+\alpha z)\right)$.) Now we can apply the preceding theorem, using the natural identification

$$
\operatorname{Pol}\left(V_{\mathbb{C}}^{+}, F\right)=\bigoplus_{k=0}^{\infty} \operatorname{Hom}\left(S^{k} V_{\mathbb{C}}^{+}, F\right)
$$

REMARK 2.3.6. - In [Ko97] T. Kobayashi announces a branching law for unitary highest weight representations with respect to certain reductive subgroups which generalizes Theorem 2.3.5.

\subsection{Spectrum of $B_{\mu}(D)$ in terms of highest weights.}

We can make Theorem 2.3.5 still more specific by describing the highest weights of the representations appearing there. Note that $K$ is a compact real form of the group $K_{\mathbb{C}}=\operatorname{Str}\left(V_{\mathbb{C}}\right)^{\alpha_{*}}$. The homomorphism

$$
\left(K_{\mathbb{C}}\right)_{o}=\operatorname{Str}\left(V_{\mathbb{C}}\right)_{o}^{\alpha_{*}} \longrightarrow \operatorname{Str}\left(V_{\mathbb{C}}^{+}\right)_{o}, \quad g \longmapsto g_{\mid V^{+}}
$$

is surjective since the map $r_{+}$considered in Proposition 1.8.1 is surjective. Thus the decomposition of $\operatorname{Pol}\left(V_{\mathbb{C}}^{+}, \mathbb{C}_{\mu}\right)$ under $K_{\mathbb{C}}$ (or under one of its covering groups) can be treated as a problem in the Jordan algebra $V^{+}$.

Now let $V_{0}$ be any Euclidian Jordan algebra (later we take $V_{0}=V^{+}$). If $V_{0}$ is simple, then according to [FK94, Th.XI.2.4] the space $\operatorname{Pol}\left(V_{0}\right)$

TOME $126-1998-\mathrm{N}^{\circ} 3$ 
of polynomials on $V_{0}$ decomposes under the standard action of $\operatorname{Str}\left(V_{0}\right)_{o}$ by $g \cdot p=p \circ g^{-1}$ as

$$
\operatorname{Pol}\left(V_{0}\right)=\bigoplus_{m \geq 0} P_{m}
$$

Here $\boldsymbol{m}=\left(m_{1}, \ldots, m_{r}\right)$ is a multi-integer $\left(r\right.$ the rank of $\left.V_{0}\right)$ with $m_{1} \geq \ldots \geq m_{r} \geq 0$, and $P_{\boldsymbol{m}}$ is a (Aut $\left(V_{0}\right)_{o}$-spherical) irreducible module of polynomials having highest weight (cf. [FK94, p. 227])

$$
-\sum_{i=1}^{r} m_{i} c_{i}^{*}
$$

with respect to the Cartan subspace $\mathfrak{a}=\oplus_{i=1}^{r} \mathbb{R} L\left(c_{i}\right)$ of $L\left(V_{0}\right)$ given by a Jordan frame $c_{1}, \ldots, c_{r}$ and to the order induced by the ordered basis $\left(L\left(c_{1}\right), \ldots, L\left(c_{r}\right)\right)$ of $\mathfrak{a}$. We denote the dual basis of $\mathfrak{a}^{*}$ by $\left(c_{1}^{*}, \ldots, c_{r}^{*}\right)$.

The module $P_{(1, \ldots, 1)}$ is one dimensional and generated by the Jordan determinant $\Delta$ of $V_{0}$. We have the relations

$$
\Delta(x)^{2 n / r}=\operatorname{Det} P(x),
$$

$\operatorname{Det} P(g x)=(\operatorname{Det} g)^{2} \operatorname{Det} P(x)$,

$$
\Delta(g x)=(\operatorname{Det} g)^{r / n} \Delta(x)
$$

for $g \in \operatorname{Str}\left(V_{0}\right)$, where $P$ is the quadratic representation of $V_{0}$. The function $\Delta$ is positive on the symmetric cone $\Omega$ of $V_{0}$, and therefore we can define $\Delta^{t}$ as a positive function on $\Omega$ for all $t \in \mathbb{R}$. In the following, we will often identify a real parameter $t$ with $(t, \ldots, t) \in \mathbb{R}^{r}$. If $\boldsymbol{m}$ is a multi-integer, we let

$$
P_{\boldsymbol{m}+t}=\left\{\Delta^{t} \cdot p ; p \in P_{\boldsymbol{m}}\right\}
$$

for general $t \in \mathbb{R}$, this is a space of functions on the symmetric cone $\Omega$, and it is a $\operatorname{Str}\left(V_{0}\right)_{0}$-module of highest weight

$$
-\sum_{i}\left(m_{i}+t\right) c_{i}^{*}
$$

For $t \in \mathbb{N}$ it is a space of polynomials coinciding with the space of polynomials defined previously. 
Theorem 2.4.1. - Assume $\alpha$ be such that $X=G / H$ is irreducible. Then for all $\mu \in \mathbb{R}$ we have the following decomposition into irreducible $K_{\infty}$-modules:

$$
\operatorname{Pol}\left(V^{+}, \mathbb{C}_{\mu}\right) \cong \bigoplus_{m \geq 0} P_{m+\mu n / r_{+}},
$$

(where notation is as above with $V_{0}=V^{+}$and $r_{+}=\operatorname{rk} V^{+}, n=\operatorname{dim} V$ ). For $\mu>\frac{1}{2}-\frac{r}{2 n}$, we have the following decomposition into irreducible $G_{\infty}$-modules:

$$
B_{\mu}(D) \cong \bigoplus_{\boldsymbol{m} \geq 0} \mathcal{H}\left(D^{-}, P_{\boldsymbol{m}+\mu n / r_{+}}\right) .
$$

The highest weight of the $G_{\infty}$-module $\mathcal{H}\left(D^{-}, P_{\boldsymbol{m}+\mu n / r_{+}}\right)$is the same as the one of the $K_{\infty}$-module $P_{m+\mu n / r_{+}}$, namely

$$
-\sum_{i}\left(m_{i}+\mu n / r_{+}\right)
$$

Proof. - We denote by Det the holomorphic character

$$
\text { Det }: K_{\mathbb{C}} \longrightarrow \mathbb{C}^{*}, \quad g \longmapsto \operatorname{Det} g .
$$

We also consider the characters

$$
\operatorname{Det}_{ \pm}: K_{\mathbb{C}} \longrightarrow \mathbb{C}^{*}, \quad g \longmapsto \operatorname{Det}\left(g_{\mid V_{\mathbb{C}}^{ \pm}}\right)
$$

then Det $=$ Det $_{+} \cdot$ Det $_{-}$. Now $K_{\mathbb{C}}$ is connected with one dimensional center; in fact, $\mathfrak{k}_{\mathbb{C}}$ is the complexification of $\mathfrak{s t r}(V)^{\alpha_{*}}$ which is (according to Proposition 1.8.3) isomorphic to $\mathfrak{s t r}\left(V^{-}\right)$; moreover $V^{-}$is simple (cf. loc. cit.), whence $\mathfrak{s t r}\left(V^{-}\right)$has a one dimensional center. Thus the center of $\mathfrak{k}_{\mathbb{C}}$ consists only of the multiples of the identity which then acts by the same scalar on $V^{+}$and $V^{-}$. Therefore

$$
\text { Det }=\operatorname{Det}_{+}^{n / n_{+}}=\left(\operatorname{Det}_{+}^{-r_{+} / n_{+}}\right)^{-n / r_{+}}
$$

so that we can calculate,

$$
\begin{aligned}
\operatorname{Pol}\left(V^{+}, \mathbb{C}_{\mu}\right) & =\operatorname{Pol}\left(V^{+}\right) \otimes \mathbb{C}_{\mu} \\
& \cong \bigoplus_{\boldsymbol{m} \geq 0} P_{\boldsymbol{m}} \otimes \operatorname{Det}^{-\mu} \\
& =\bigoplus_{\boldsymbol{m} \geq 0} P_{\boldsymbol{m}} \otimes\left(\operatorname{Det}_{+}^{-r_{+} / n_{+}}\right)^{-\mu n / r_{+}}=\bigoplus_{\boldsymbol{m} \geq 0} P_{\boldsymbol{m}+\mu n / r^{+}}
\end{aligned}
$$

is the decomposition under $\operatorname{Str}\left(V^{+}\right)_{o}$, under $\operatorname{Str}(V)_{o}^{\alpha_{*}}$ (because of the surjectivity of (2.4)), and thus also under $K_{\mathbb{C}}$ and $K$.

$$
\text { TOME } 126-1998-\mathrm{N}^{\circ} 3
$$


The statement about the decomposition of $B_{\mu}(D)$ now follows from Theorem 2.3.5, and the formula for the highest weight w.r.t. $K_{\infty}$ is an immediate consequence of formula (HW) above. The remaining statement about the corresponding highest weights w.r.t. $G_{\infty}$ is a general fact on holomorphically induced representations, using the fact that $\mathfrak{a}_{\mathbb{C}}$ is a Cartan subspace both in $\mathfrak{k}_{\mathbb{C}}$ and in $\mathfrak{g}_{\mathbb{C}}(c f .[$ Ach96,Th. 3.7]).

REMARKS 2.4.2.

(i) Note that $\frac{n}{r_{+}}=\frac{2 n}{r}$ if $\alpha$ is split and $\frac{n}{r_{+}}=\frac{n}{r}$ if $\alpha$ is non-split.

(ii) If $\alpha$ is a Peirce involution, then $V^{+}=V_{1} \oplus V_{-1}$ is a direct sum of two simple algebras, and the preceding theorem cannot be applied. In fact, for the explicit decomposition of $\operatorname{Pol}\left(V_{1} \oplus V_{-1}\right) \cong \operatorname{Pol}\left(V_{1}\right) \otimes \operatorname{Pol}\left(V_{-1}\right)$ in terms of the ones of $\operatorname{Pol}\left(V_{1}\right)$ and $\operatorname{Pol}\left(V_{-1}\right)$ one needs a Clebsch-Gordan formula. Then one could apply similar arguments as in the preceding proof.

\section{Comparison of Hardy spaces}

\subsection{The Hardy space associated to $\Xi$.}

We keep the notation and hypotheses from Section 2. Following [HOØ91], we define a Hardy space $H^{2}(\Xi)$ associated to the compactly causal Makarevič space $X$ as the space of holomorphic functions $f$ on $\Xi$ having finite Hardy space norm

$$
\|f\|_{H^{2}(\Xi)}^{2}:=\sup _{\gamma \in \Gamma^{-1}}\|\gamma \cdot f\|_{L^{2}(X)}^{2}
$$

where $\Gamma=G_{\mathbb{C}} \cap S(D) \subseteq G_{\mathbb{C}}$ is the semigroup introduced in Proposition 1.4.1; it acts on the space of holomorphic functions $f: \Xi \rightarrow \mathbb{C}$ by $\gamma \cdot f:=f \circ \gamma^{-1}$. Since $\gamma \cdot f$ is holomorphic on a neighborhood of $\Xi$, it is continuous on $X$, and

$$
\|\gamma \cdot f\|_{L^{2}(X)}^{2}:=\int_{X}\left|f\left(\gamma^{-1} x\right)\right|^{2} \mathrm{~d} x
$$

is defined. We recall the following general results on $H^{2}(\Xi)$ from [HOØ91]:

THEOREM 3.1.1.

(i) Equation (3.1) defines a Hilbert space norm on $H^{2}(\Xi)$.

(ii) The semigroup $\Gamma^{-1}$ acts by contractions on $H^{2}(\Xi)$ via $(\gamma, f) \mapsto \gamma \cdot f$.

BULLETIN DE LA SOCIÉTÉ MATHÉMATIQUE DE FRANCE 
(iii) There is a G-equivariant isometry $b=b_{\Xi}: H^{2}(\Xi) \rightarrow L^{2}(X)$ defined by $b_{\Xi}(f)=\lim \left(\gamma_{j} \cdot f\right)$ for any sequence $\gamma_{j} \in \Gamma^{-1}$ converging to $1 \in G$. Here the action of $G$ on $L^{2}(X)$ is the left regular one.

(iv) Set $W_{0}:=-\widetilde{W}$ and suppose that $X \in i W_{0}$. Then $X$ acts on $b_{\Xi}\left(H^{2}(\Xi)\right)$ via the regular representation as a negative operator. Conversely, if $\mathcal{H} \subseteq L^{2}(X)$ is a closed subspace on which all the $X \in i W_{0}$ act as negative operators, i.e. $\mathcal{H}$ is $W_{0}$-dissipative, then $\mathcal{H} \subseteq b \Xi\left(H^{2}(\Xi)\right)$.

(v) The Hilbert space $H^{2}(\Xi)$ admits a reproducing kernel $K: \Xi \times \Xi \rightarrow \mathbb{C}$ which is holomorphic in the first and antiholomorphic in the second variable.

Since the Hardy space $H^{2}(\Xi)$ is defined by integration over $X$, we will need the following result about the $G$-invariant measure on $X$. Let us denote by $d \sigma$ the $U$-invariant normalized measure on $\Sigma$.

Proposition 3.1.2. - The density of the G-invariant measure on the open dense set $X \subset \Sigma$ with respect to $d \sigma$ is given by the function

$$
\left|f_{\alpha}(u)\right|^{-\frac{1}{2}}, \quad u \in X
$$

Proof. - We use the corresponding result for the Cayley transformed realization: by [Be98, Cor. 2.4.2], the invariant measure on the space $X^{(-\alpha)}=C(X)$ is given by

$$
\left|\operatorname{Det} B_{-\alpha}(x, x)\right|^{-\frac{1}{2}} \mathrm{~d} \lambda
$$

(where $\mathrm{d} \lambda$ is Lebesgue measure on $V$ ) and the invariant measure on $V^{c}=X^{\left(-\mathrm{id}_{V}\right)}$ by

$$
\left|\operatorname{Det} B_{-\mathrm{id}}(x, x)\right|^{-\frac{1}{2}} \mathrm{~d} \lambda \text {. }
$$

In both formulas, Det $A$ denotes the determinant of an endomorphism $A$ of $V$; it is the same as the determinant of its $\mathbb{C}$-linear extension $A_{\mathbb{C}}$ taken over $\mathbb{C}$ (since both have the same matrix). By Cayley transform, the density we are looking for is given by

$$
\frac{\left|\operatorname{Det} B_{-\alpha}(C x, C x)\right|^{-\frac{1}{2}}}{\left|\operatorname{Det} B_{-\mathrm{id}}(C x, C x)\right|^{-\frac{1}{2}}}
$$

The calculation given in the proof of [Be98, Th. 2.1.1] together with the fact that $|\operatorname{Det} P(u)|=1$ for all $u \in \Sigma$ shows that the quotient equals

$$
|\operatorname{Det} P(z+\alpha z)|^{-\frac{1}{2}}, \quad z=C(x) \in C(X) .
$$

TOME $126-1998-\mathrm{N}^{\circ} 3$ 


\subsection{Embedding of $H^{2}(D)$ into $H^{2}(\Xi)$ (proof of Theorem 3).}

Recall the definition of the Hardy space $H^{2}(D)$ and its reproducing kernel from Equations (0.10) and (0.11). We are now ready to give a precise formulation for Theorem 3.

Theorem 3.2.1. - Assume that $\frac{1}{4}$ is an admissible parameter, i.e. $f_{\alpha}$ has a holomorphic fourth root.

(i) We have a norm preserving inclusion of function spaces

$$
B_{\frac{1}{2}, \frac{1}{2}}(\Xi) \subset H^{2}(\Xi)
$$

(ii) The map

$$
H^{2}(D)=B_{\frac{1}{2}}(D) \longrightarrow B_{\frac{1}{2}, \frac{1}{2}}(\Xi) \subset H^{2}(\Xi), \quad f \longmapsto f_{\alpha}^{\frac{1}{4}} \cdot f
$$

is an isometric and $G$-equivariant imbedding.

Proof.

(i) Proposition 2.2.4 shows that $\Gamma^{-1}$ acts by contractions in $B_{\frac{1}{2}}^{2}(D)$ and thus, by $G$-isomorphy, also in the space $B_{\frac{1}{2}, \frac{1}{2}}(\Xi)$. Recall further that the classical Hardy space $H^{2}(D)$ also admits an isometric embedding $b_{D}: H^{2}(D) \rightarrow L^{2}(\Sigma), f \mapsto \lim _{t \rightarrow 1} t^{-1}$ id $\cdot f(c f .[\mathrm{FK} 94$, p. 270]). Using this and Proposition 3.1.2, we have for all $f \in B_{\frac{1}{2}, \frac{1}{2}}(\Xi)$

$$
\begin{aligned}
\|f\|_{B_{\frac{1}{2}, \frac{1}{2}}(\Xi)} & =\sup _{\gamma \in \Gamma^{-1}}\|\gamma \cdot f\|_{B_{\frac{1}{2}, \frac{1}{2}}(\Xi)} \\
& =\sup _{\gamma \in \Gamma^{-1}}\left\|f_{\alpha}^{-\frac{1}{4}}(\gamma \cdot f)\right\|_{B_{\frac{1}{2}, 0}(\Xi)} \\
& =\sup _{\gamma \in \Gamma^{-1}}\left\|f_{\alpha}^{-\frac{1}{4}}(\gamma \cdot f)\right\|_{H^{2}(D)} \\
& \left.=\lim _{t \rightarrow 1} \sup _{\gamma \in \Gamma^{-1}}\left\|t^{-1} \mathrm{id} \cdot\left(f_{\alpha}^{-\frac{1}{4}}(\gamma \cdot f)\right)\right\|_{H^{2}(D)}\right) \\
& =\sup _{\gamma \in \Gamma^{-1}}\left\|f_{\alpha}^{-\frac{1}{4}}(\gamma \cdot f)\right\|_{L^{2}(\Sigma)} \\
& =\sup _{\gamma \in \Gamma^{-1}}\|\gamma \cdot f\|_{L^{2}(X)}=\|f\|_{H^{2}(\Xi)} .
\end{aligned}
$$

This proves part (i). Part (ii) follows by combining part (i) and Proposition 2.1.3.

\subsection{Criterion for equality (proof of Theorem 4).}

Recall the description of the spectrum of $H^{2}(\Xi)$ in terms of highest weight representations from [HOØ91, Th. 3.3 and Th. 3.4] and [OØ91, Th. 5.2]. 
Theorem 3.3.1. - Suppose that $X$ is irreducible.

(i) $H^{2}(\Xi)$ is a multiplicity free direct sum of unitary highest weight modules.

(ii) The highest weight representations occurring in $H^{2}(\Xi)$ admit $H$ invariant distribution vectors so that the minimal $K$-types are $K \cap H$ spherical and the corresponding highest weights can be viewed as elements of $\mathfrak{a}^{*}$.

(iii) An element of $\lambda \in i \mathfrak{a}^{*}$ occurs as a highest weight of an irreducible subrepresentation of $H^{2}(\Xi)$ if and only the following conditions are satisfied:

(1) the $K$-module with highest weight $\lambda$ is $K \cap H$-spherical,

(2) $\lambda(i X) \leq 0$ for all $X \in W \cap \mathfrak{a}=-W_{0} \cap \mathfrak{a}$,

(3) $(\lambda+\rho \mid \beta)<0$ for all $\beta \in \Delta_{1}$.

The generalization of the Cartan-Helgason theorem given by Takeuchi in [Ta73, Th. 2.4] allows a characterization of the property of the $K$ module with highest weight $\lambda$ to be $K \cap H$-spherical. The integrality condition on $\lambda$ given in that theorem refers to the lattice

$$
L:=\{Z \in \mathfrak{a} ; \exp (Z) i e=i e\}
$$

If $e_{1}, \ldots, e_{r_{+}}$is a Jordan frame in $V^{+}$, then

$$
L=2 \pi i \sum_{j=1}^{r_{+}} \mathbb{Z} L\left(e_{j}\right) ;
$$

this is immediate from the equation $\exp \left(L\left(\sum_{j} a_{j} e_{j}\right)\right) i e=i \sum_{j} e^{a_{j}} e_{j}$. If now

$$
\lambda=\sum_{j=1}^{r_{+}} m_{j} e_{j}^{*},
$$

the integrality condition from Takeuchi's Cartan-Helgason theorem simply is

$$
m_{j} \in \mathbb{Z}, \quad \forall j=1, \ldots, r_{+} .
$$

Of course the corresponding dominance condition depends on the choices of $\Delta_{0}^{+}$made in Section 1.7.

Theorem 3.3.2. - Assume that $X$ is irreducible and that $\frac{1}{4}$ is an admissible parameter.

(i) If $\alpha$ is non-split, then $B_{\frac{1}{2}, \frac{1}{2}}(\Xi)=H^{2}(\Xi)$. These are the cases I.3 $(\operatorname{Sp}(2 k, \mathbb{R}) / \operatorname{Sp}(k, \mathbb{C}))$, II.2 $(\operatorname{Sp}(k, \mathbb{R}))$, IV.3 (Hyperboloids) and Cayley type from the classification in Table 1.5.1.

TOME $126-1998-\mathrm{N}^{\circ} 3$ 
(ii) If $\alpha$ is split, then $B_{\frac{1}{2}, \frac{1}{2}}(\Xi)$ is a proper subspace of $H^{2}(\Xi)$, and the quotient $H^{2}(\Xi) / B_{\frac{1}{2}, \frac{1}{2}}(\Xi)$ decomposes as a direct sum of irreducible $G$-modules with highest weights $\lambda=-\sum_{j=1}^{r} m_{j} e_{j}^{*}$ as follows:

$$
m_{1} \geq \cdots \geq m_{r}, \quad m_{i} \in \mathbb{Z}^{+}, \quad \frac{n}{2 r}>m_{r}>\frac{n}{2 r}-\frac{d+1}{2} .
$$

These are the cases I.2 $\left(\mathrm{SO}^{*}(2 m) / \mathrm{SO}(m, \mathbb{C}) ; d=2\right)$, III.2 $\left(\mathrm{SO}^{*}(2 m)\right.$; $d=4)$ and V.2 $(d=8)$.

Proof. - We have to specify the conditions of Theorem 3.3 .1 (iii) in terms of the Jordan data from Section 1.7. If we let

$$
\lambda=-\sum_{i=1}^{r_{+}} m_{i} e_{i}^{*}
$$

then the description of $\Delta_{0}^{+}$shows that condition (1) from Theorem 3.3.1 can be written

$$
m_{1} \geq \cdots \geq m_{r}, \quad m_{i} \in \mathbb{Z} .
$$

Since $\mathfrak{a} \cap W=-\sum_{i=1}^{r_{+}} \mathbb{R}^{+} e_{i}$, the cone admissibility condition (2) can be written

$$
\lambda=-\sum_{i=1}^{r_{+}} m_{i} e_{i}^{*}, \quad \forall i, m_{i}>0 .
$$

The conditions (1) and (2) together have been written earlier in the form $\boldsymbol{m} \geq 0$. The Harish-Chandra condition (3) has to be made explicit by using the structural data from Section 1.7.

- $\alpha$ split. - The condition $(\rho-\boldsymbol{m} \mid \beta)<0$ for all $\beta \in \Delta_{1}$ is equivalent to

$$
m_{j}+m_{k}>\rho_{j}+\rho_{k} \quad \forall j \neq k,
$$

where $\rho=\sum_{j} \rho_{j} e_{j}^{*}$. Since $m_{j}+m_{k} \geq 2 m_{r}$ and $\rho_{j}+\rho_{k} \leq \rho_{r}+\rho_{r-1}=$ $\frac{1}{4} d(2 r-3)$, this condition is equivalent to

$$
m_{r}>\frac{d}{2}(2 r-3)=\frac{n}{2 r}-\frac{d+1}{2} .
$$

- $\alpha$ non-split. - The condition $(\rho-\boldsymbol{m} \mid \beta)<0$ for all $\beta \in \Delta_{1}$ is equivalent to

$$
m_{j}>\rho_{j} \quad \forall j
$$

BULLETIN DE LA SOCIÉTÉ MATHÉMATIQUE DE FRANCE 
In the Cayley-type case the Harish-Chandra condition is equivalent to

$$
m_{r_{+}}>\rho_{r_{+}}=\frac{1}{2}+\frac{d}{2}\left(r_{+}-1\right)=\frac{n_{+}}{r_{+}}-\frac{1}{2}=\frac{n}{r}-\frac{1}{2} .
$$

Since $n / r$ is either an integer or a half-integer, this is equivalent to $m_{r_{+}} \geq n / r$.

In the matrix cases the Harish-Chandra condition is equivalent to

$$
m_{r_{+}}>\rho_{r_{+}}=d\left(r_{+}-1\right)+\frac{d+1}{2}=\frac{d(r-1)}{2}+\frac{1}{2}=\frac{n}{r}-\frac{1}{2} .
$$

As above, this is seen to be equivalent to $m_{r_{+}} \geq n / r$.

In the case of Type IV.3 we have $\rho=\frac{1}{2}(n-1) e^{*}$; thus the HarishChandra condition is equivalent to

$$
n_{r_{+}}>\rho_{r_{+}}=\frac{n-1}{2}=\frac{n}{r}-\frac{1}{2}
$$

and again this is equivalent to $m_{r_{+}} \geq n / r$.

Now we can compare conditions (1)-(3) of Theorem 3.3.1 (iii) with the description of the spectrum of $B_{\frac{1}{2}, \frac{1}{2}}(\Xi) \cong B_{\frac{1}{2}}(D)$ (Theorem 2.4.1): the spectrum of $B_{\frac{1}{2}}(D)$ is given by the condition $\boldsymbol{m} \geq 0$ and $m_{r} \geq n /\left(2 r_{+}\right)$. In the non-split case the last condition is equivalent to $m_{r} \geq n / r$. Since the Harish-Chandra condition was also equivalent to this condition, the spectra of $B_{\frac{1}{2}, \frac{1}{2}}(\Xi)$ and $H^{2}(\Xi)$ are the same, and since according to Th. 3.3.1 (i) we have multiplicity one in $H^{2}(\Xi)$, both spaces are actually equal. In the split case the spectrum of $B_{\frac{1}{2}}(D)$ is given by the conditions $\boldsymbol{m} \geq 0$ and $m_{r} \geq n /(2 r)$. Since in these cases $d \geq 2$, this does not coincide with the conditions (1)-(3) given above, the difference of both conditions being precisely the set of parameters given in the theorem.

Remark 3.3.3. - If $\alpha$ is a Peirce involution, it is more difficult to get precise results. However, going through the arguments leading to Theorem 3.3.2, one gets rough estimates indicating that in these cases again $B_{\frac{1}{2}, \frac{1}{2}}(D)$ is a proper subspace of $H^{2}(\Xi)$.

\subsection{The compact case.}

We consider the case $\alpha=\operatorname{id}_{V}: X=\Sigma$ is the Shilov boundary of $D$, and $\Xi=\{z \in D ; \operatorname{det} z \neq 0\}=D \cap \Omega_{\mathbb{C}}$. Note that Theorem 3.3.2 does not apply in this case since $X$ is not irreducible. The following result, due to Lassalle (see [La85, Th. 8]), replaces Theorem 3.3.2 in the compact case.

TOME $126-1998-\mathrm{N}^{\circ} 3$ 
Proposition 3.4.1. $-H^{2}(\Xi)=H^{2}(D)$.

\section{Proof.}

Part "つ". - Clearly a function holomorphic on $D$ is holomorphic on $\Xi$, and an element of $H^{2}(D)$ satisfies also the Hardy condition of $H^{2}(\Xi)$ because according to Proposition 2.2.4, $S(D)^{-1}$ acts by contractions on $H^{2}(D)$.

Part " $\subset$ ". - It suffices to show that elements of $H^{2}(\Xi)$ are actually holomorphic on $D$ for then the Hardy condition of $H^{2}(\Xi)$ clearly implies the one of $H^{2}(D)$. (The supremum is taken over the semigroup $] 0,1[\subset$ $\Gamma(C)=\left\{g \in \operatorname{Str}\left(V_{\mathbb{C}}\right) ; g(\Xi) \subset \Xi\right\}$.) According to [FK94, Ch. XII], every holomorphic function $f$ on $\Xi$ can be developped in a Laurent series

$$
f(z)=\sum_{\boldsymbol{m} \in \mathbb{Z}_{+}^{r}} a_{\boldsymbol{m}} p_{\boldsymbol{m}}(z)
$$

where $p_{\boldsymbol{m}}$ belongs to the space $P_{\boldsymbol{m}}$ defined in [FK94], p. 245. The elements of this space are certain rational functions which are homogeous of degree $\sum_{i=1}^{r} m_{i}$. Since

$$
\sup _{0<r<1} \int_{\Sigma}\left|p_{\boldsymbol{m}}(r u)\right|^{2} \mathrm{~d} \sigma(u)=\left(\sup _{0<r<1} r^{\Sigma m_{i}}\right) \int_{\Sigma}\left|p_{\boldsymbol{m}}(r u)\right|^{2}
$$

is finite if and only if $\sum m_{i} \geq 0$, only terms satisfying this condition appear in the Laurent series of $f$ which is thus actually a Taylor series converging on $D$.

Corollary 3.4.2. - The reproducing kernel of $H^{2}(\Xi)$ is given by $K(z, w)=\operatorname{Det} B(z, \bar{w})^{-\frac{1}{2}}$.

Corollary 3.4.3. - In the compact case, the inclusion $B_{\frac{1}{2}}(\Xi) \subset H^{2}(\Xi)$ is strict, and the Bergman kernel associated to $\Xi$ is not the square of the Cauchy kernel.

Proof. - The first statement follows from the second, and this is proved by remarking that the Bergman kernel for $\Xi$ is given by

$$
K^{(1,1)}(z, w)=\operatorname{Det} P(z)^{\frac{1}{2}} \operatorname{Det} B(z, \bar{w})^{-1} \operatorname{Det} P(\bar{w})^{\frac{1}{2}}
$$

which is not the square of $K$.

The results of the last corollary make appear the equality in Proposition 3.4.1 rather "non-canonical". It is difficult to give a reasonable interpretation of this equality. It can be proved that for any $\alpha$ the inclusion $H^{2}(\Xi) \subset H^{2}(D)$ holds; thus the space $H^{2}(D)$ appears as an "upper bound" which is reached in the compact case. 


\section{Open problems}

At this stage there are two classes of open problems:

Problem 4.1. - A more conceptual definition of the spaces $B_{\mu, \lambda}^{2}(\Xi)$ has to be made in the framework of Hilbert spaces of sections of vector bundles; then the spaces $B_{\mu, 2 k}^{2}(\Xi)$ for various admissible parameters $k$ are all interpreted as spaces of sections of the same line bundle. The groundwork for such a theory is laid in the note [BH97]. However, the definition of Hardy spaces as spaces of sections of line bundles (of half-forms, to be more precise) is more delicate than the definition of Bergman spaces of sections. For example, topological problems come in, related to the problem of existence of holomorphic fourth roots of $f_{\alpha}$. But only in this way it is possible to get an interpretation of the "inclusion" of the classical Hardy space in the non-classical one as a natural inclusion of invariantly defined Hardy spaces, and at the same time to make definitions independent of the special realization chosen. The desire for having such an invariant description arises already in the case of the classical Hardy spaces: in fact, the classical Hardy space $H^{2}(D)$ and the Hardy space $H^{2}\left(T_{\Omega}\right)$ of the tube $T_{\Omega}$ equivalent to $D$ (see [FK94, p. 178]) are isomorphic, but this is not at all clear from the definitions (it can be deduced by comparing the reproducing kernels). Therefore one would like to understand $H^{2}(D)$ and $H^{2}\left(T_{\Omega}\right)$ as different aspects of one invariantly defined object. Then, as shows the present work, the invariantly defined Hardy space of $\Xi$ sometimes is equal to the one of $D$, but sometimes it is strictly bigger. One would like to have a geometric understanding of Theorem 4 in this context.

Problem 4.2. - One wants to find a formula for the Cauchy-Szegö kernel in the remaining cases, where one does not have equality of classical and non-commutative Hardy spaces. There is one case in which this is easy, namely the compact case; $c f$. Section 3.4 . In the intermediate cases it seems to be rather difficult to find a "simple" formula for the CauchySzegö kernel ( $c f$. [KØ96] for the case $X=\mathrm{U}(1,1)$ ). Moreover, there are a few compactly causal spaces which cannot be realized using Jordan algebras ( $c f$. Remark 1.5.2), and for these literally nothing seems to be known. 


\section{BIBLIOGRAPHY}

[Ach96] Аснав (D.). - Représentations C-dissipatives irréductibles. - Journées 'Programme de Gelfand-Gindikin', Paris, 1996.

[Be96] Bertram (W.). - On some Causal and Conformal Groups, J. Lie Theory, t. 6, 1996, p. 215-244.

[Be98] Bertram (W.). - Algebraic Structures of Makarevič Spaces I, Transformation Groups, t. 3, 1998, p. 3-32.

[BH97] Bertram (W.), Hilgert (J.). - Reproducing Kernels on Vector Bundles. - Preprint, Clausthal, 1997.

[BO98] Betten (F.), Ólafsson (G.). - Causal compactifications and Hardy spaces for spaces of Hermitian type. - Submitted.

[Cha98] Chadui (M.). - Espace de Hardy d'un espace symétrique de type Cayley, Ann. Inst. Fourier, t. 48, 1998, p. 97-132.

[Chab90] Снават (B.). - Introduction à l'analyse complexe, vol. 2. - Éditions MIR, Moscou, 1990.

[FK94] Faraut (J.), Korányi (A.). - Analysis on symmetric cones. Clarendon Press, Oxford, 1994.

[FO95] Faraut (J.), Ólafsson (G.). - Causal semi-simple symmetric spaces, the geometry and harmonic analysis, in 'Semigroups in Algebra, Geometry and Analysis'. - Hofmann, Lawson, Vinberg, eds.; de Gruyter, Berlin, 1995.

[Hw69] Helwig (K.H.). - Involutionen von Jordan-Algebren, Man. Math., t. 1, 1969, p. 211-229.

[HO96] Hilgert (J.), Ólafsson (G.). - Causal Symmetric Spaces Geometry and Harmonic Analysis, Perspectives in Math., t. 18, Academic Press, San Diego, 1996.

[HOØ91] Hilgert (J.), Ólafsson (G.), Ørsted (B.). - Hardy spaces on affine symmetric spaces, J. reine angew. Math., t. 415, 1991, p. 189-218.

[JV79] JAKobSEn (H.P.), Vergne (M.). - Restriction and Expansion of Holomorphic Representations, J. Functional Analysis, t. 34, 1979, p. 29-53.

[Ko68] Kobayashi (S.). - Irreducibility of certain unitary representations, J. Math. Soc. Japan, t. 20, 1968, p. 638-642.

[Ko97] KoBAyashi (T.). - Multiplicity free theorem in branching problems of unitary highest weight modules, in 'Proceedings of the annual meeting of Representation Theory at Saga 1997'. - K. Mimachi, ed., p. 9-17.

[KØ96] Koufany (K.), Ørsted (B.). - Function spaces on the Olshanski semi-group and the Gelfand-Gindikin program, Ann. Inst. Fourier, t. 46, 1996, p. 689-722. 
[KØ97] Koufany (K.), ØRSTED (B.).--Hardy spaces on two-sheeted covering semigroups, J. Lie theory, t. 7, 1997, p. 245-267.

[Kr97] Kröтz (B.). - On Hardy and Bergman spaces on complex Olshanskii semigroups. - Submitted.

[La85] Lassalle (M.). - L'espace de Hardy d'un domaine de Reinhard généralisé, J. Funct. Anal., t. 60, 1985, p. 309-340.

[Lo77] Loos (O.). - Bounded symmetric domains and Jordan pairs. Lecture Notes, Irvine, 1977.

[Ma73] MakAREvič (B.O.). - Open symmetric orbits of reductive groups in symmetric $R$-spaces, Math. USSR Sbornik, t. 20, 1973, p. 406-418.

[Mo97] Molchanov (V.). - Holomorphic Disrete Series for Hyperboloids of Hermitian Type, J. Funct. Anal., t. 147, 1997, p. 26-50.

[Ne98] NeEb (K.H.). - Holomorphy and Convexity in Lie Theory. - de Gruyter, to appear.

[OØ91] Ólafasson (G.), ØRSTed (B.). - The holomorphic discrete series of an affine symmetric space and representations with reproducing kernel, Trans. Am. Math. Soc., t. 326, 1991, p. 385-405.

[OØ98] Ólafsson (G.), Ørsted (B.). - Causal compactifications and Hardy Spaces, Trans. AMS, to appear.

[Pe96] Pevsner (M.). - Espace de Bergman d'un semi-groupe complexe, Comptes Rendus Ac. Sci. Paris, Série 1, t. 322, 1996, p. 635-640.

[Sa80] SATAKe (I.).--Algebraic structures of symmetric domains. - Iwanami Shoten, Princeton, 1980.

[Ta73] Takeuchi (M.). - Polynomial representations associated with symmetric bounded domains, Osaka J. Math., t. 10, 1973, p. 441-475. 